\title{
A porous fibrous hyperelastic damage model for human periodontal ligament: Application of a micro-computerized tomography finite element model.
}

J. Ortún-Terrazas ${ }^{\text {a }}$, J. Cegoñino ${ }^{\text {a }}$, U. Santana-Penín ${ }^{\text {b }}$, U. Santana-Mora ${ }^{\text {b }}$, A. Pérez del Palomar ${ }^{\text {a }}$

1 Group of Biomaterials, Aragon Institute of Engineering Research (I3A), Department of Mechanical Engineering, University of Zaragoza, Zaragoza, Spain

${ }^{2}$ School of Dentistry, Faculty of Medicine and Odontology, Santiago de Compostela University, Santiago de Compostela, Spain

Correspondence to: Javier Ortún-Terrazas, Department of Mechanical Engineering, University of Zaragoza, Spain, María de Luna nº3, Betancourt building, 50018 Zaragoza, Spain. E-mail: javierortun@unizar.es

\section{Abstract}

The periodontal ligament (PDL) is a soft biological tissue that connects the tooth with the trabecular bone of the mandible. It plays a key role in load transmission and is primarily responsible for bone resorption and most common periodontal diseases. Although several numerical studies have analysed the biomechanical response of the PDL, most did not consider its porous fibrous structure, and only a few analysed damage to the PDL. This study presents an innovative numerical formulation of a porous fibrous hyperelastic damage material model for the PDL. The model considers two separate softening phenomena: fibre alignment during loading and fibre rupture. The parameters for the material model characterization were fitted using experimental data from the literature. Furthermore, the experimental tests used for characterization were computationally modelled to verify the material parameters. A finite element model of a portion of a human mandible, obtained by micro-computerized tomography, was developed, and the proposed constitutive model was implemented for the PDL. Our results confirm that damage to the PDL may occur mainly due to overpressure of the interstitial fluid, while large forces must be applied to damage the PDL fibrous network. Moreover, this study clarifies some aspects of the relationship between PDL damage and the bone remodelling process.

Keywords: Periodontal ligament, Damage model, Fibrous network, Micro-computed tomography, Mullins effect, Porous elasticity.

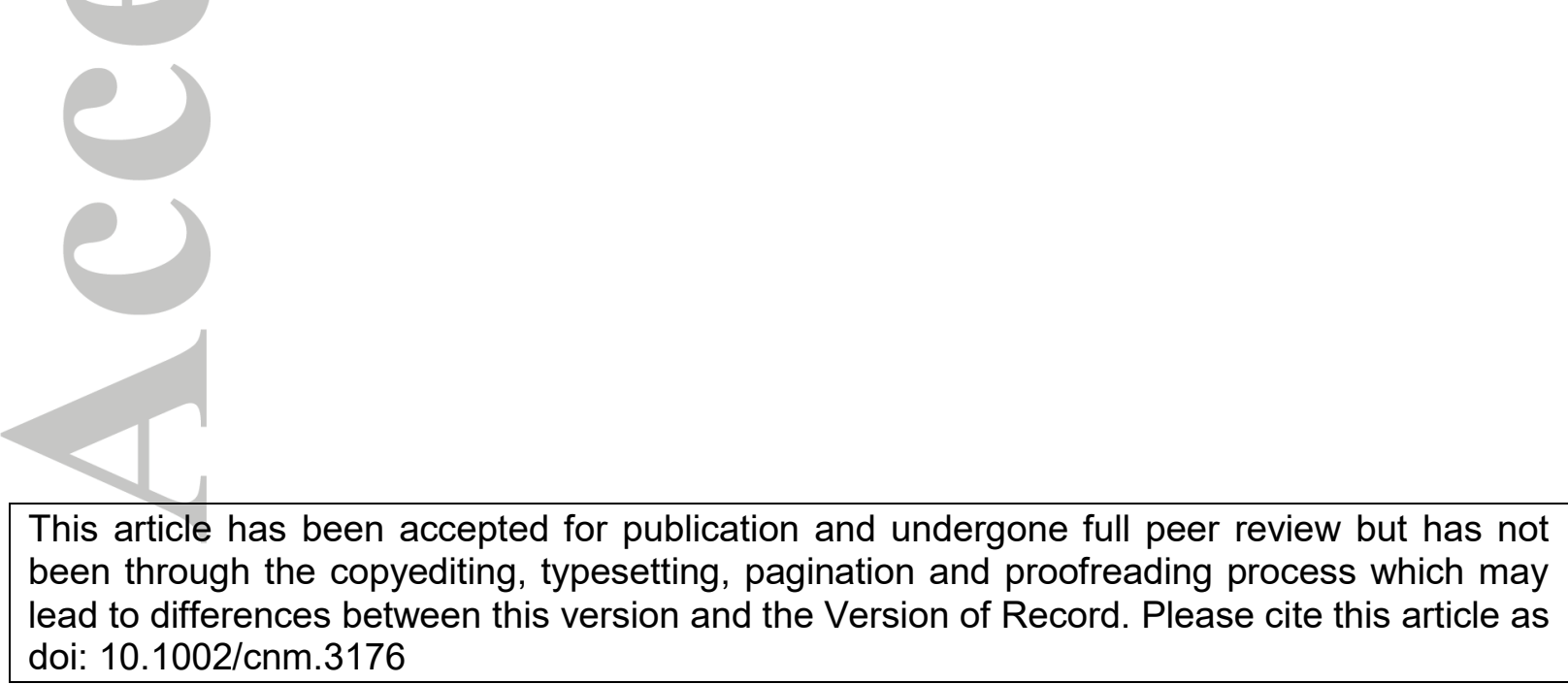

This article is protected by copyright. All rights reserved. 


\section{1 | INTRODUCTION}

The periodontal ligament (PDL) is a soft connective tissue that absorbs and distributes chewing loads. A healthy PDL contributes to homogeneous transmission of loads to the trabecular bone of the mandible. However, several mechanical factors may affect periodontal health and cause destruction of the connective matrix, loss of fibrous attachment or bone remodelling. ${ }^{1}$

Anatomically, the human periodontium is composed of the gum $(\mathrm{G})$, trabecular bone (TB), cortical bone (CB), the tooth and the PDL. The tooth is mainly formed of enamel (E), dentin (D), pulp (P) and cementum root (CR) (Figure 1A). The gum is the main responsible for periodontal diseases produced by bacterial action, although the gum is less relevant from a biomechanical point of view. ${ }^{2}$ In contrast, dentin, bone and the PDL are the main agents for load transmission, and some of the most common periodontal diseases are related to an abnormal mechanical role of these elements. ${ }^{3}$

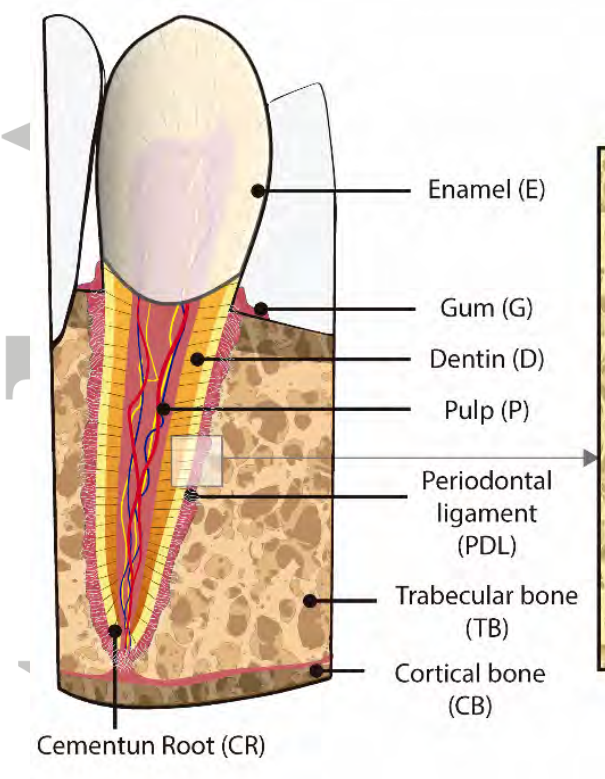

(A)

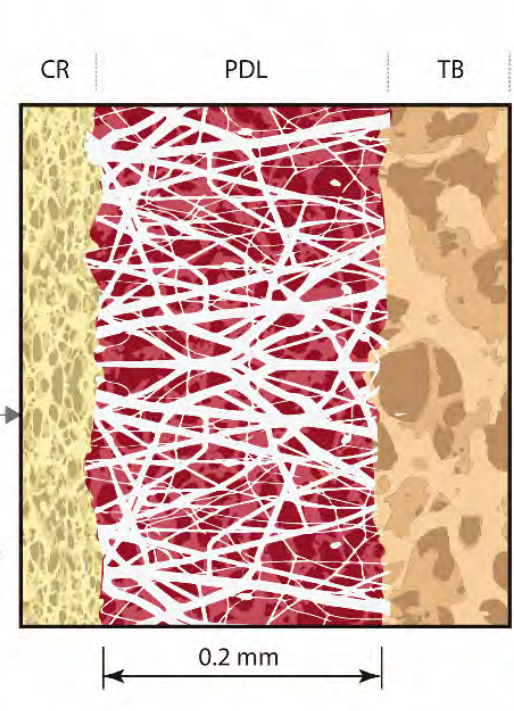

(B)

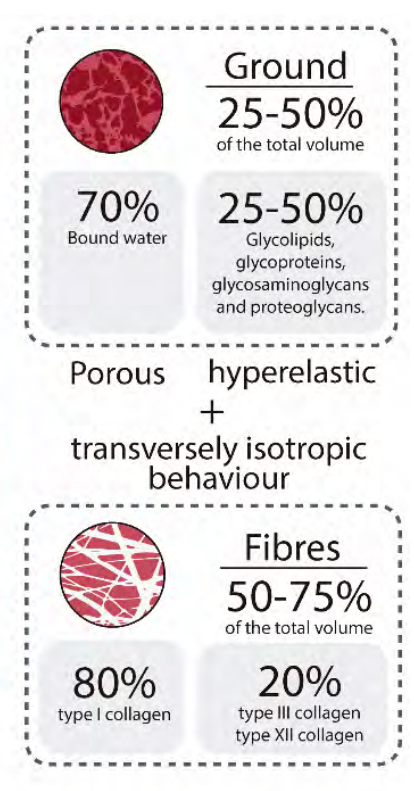
FIGURE 1 (A) Schematic of human canine tooth anatomy; (B) Details of the periodontal ligament structure and
relationship between its composition and a porous hyperelastic and transversely isotropic material model.

Biomechanically, the PDL is a soft biological tissue that can be considered a porous vasculature solid with a highly structured collagen network. ${ }^{4-6}$ Approximately $50-75 \%$ of the PDL volume is formed by collagen fibres, ${ }^{7}$ primarily type $\mathrm{I}^{7}{ }^{\mathrm{III}}{ }^{8}$ and XII fibres (Figure 1B). ${ }^{9}$ The fibres are primarily responsible for the differences observed in PDL reactions under tensile force. On the other hand, the high vascular density of the PDL confers the tissue a viscoelastic behaviour, which has been extensively studied in the literature. ${ }^{10-12}$ Thus, the tissue behaves as an incompressible material under fast strains and as a highly compressible material under slow strains due to this viscoelastic phenomenon. ${ }^{5,13-15}$ Neglecting the fibrous and time-dependent behaviour of the PDL is a typical source of error in experimental studies found in the literature. ${ }^{14,16,17}$ Thus, large variations in both the elastic modulus (0.01-1750 $\mathrm{MPa})$ and Poisson's ratio (0.28-0.49) have been reported. ${ }^{17}$ Furthermore, these values are 
often experimentally obtained using rodent PDL samples, ${ }^{17-19}$ which have a masticatory system that is extremely different from that in humans. ${ }^{20}$ Fortunately, the number of studies that used porcine or bovine samples has increased in recent years. Moreover, the experimental protocol is another common source of error. ${ }^{17}$

Due to the difficulty obtaining accurate experimental measurements, finite element (FE) analysis has been extensively used. Some authors ${ }^{21-24}$ have recently used FE models obtained by micro-computerized tomography (microCT). The authors of these studies assert the importance of considering the irregular geometry of the PDL to obtain reliable results. Some FE studies use isotropic hyperelastic or elastic properties ${ }^{23,25,26}$ for the ligament or viscoelastic material models. ${ }^{27,28}$ Instead of defining the PDL as an isotropic tissue without fluid coupling, we considered the porous and fibrous components of the PDL in our previous study. ${ }^{24}$ Thus, a complex material model that takes into account different behaviours for tensile and compressive effects was developed. However, damage to the PDL tissue has been poorly analysed, and our previous study ${ }^{24}$ only explained the PDL response under a nontraumatic loading scenario. The visco-hyperelastic damage model proposed by Natali et al ${ }^{28,16}$ is one of the most comprehensive studies of PDL damage. They proposed a material model that considers anisotropy, an almost-incompressible behaviour, time dependence and the damage phenomenon for large strains. Nevertheless, the softening phenomena due to repetitive loads or fibrous network rupture remain unknown. The experimental results reported by Natali et al ${ }^{29}$ show a softening phenomenon of the tissue under load repetition that may be caused by rearrangement of fibres. ${ }^{16,26,30}$ In the non-loading state, the fibrous network is partially disorganized with interweaved collagen fibres. ${ }^{31}$ This organization leads to a stiffer behaviour of the matrix than if the fibres were not interconnected. When the tissue is stretched, the local movement of the collagen fibres causes the fibrous network to become untangled, resulting in a softening phenomenon ${ }^{31}$ that has been observed in other soft tissues, such as arteries, ${ }^{32}$ veins, ${ }^{33}$ vaginal tissues, ${ }^{34}$ oesophageal tissues, ${ }^{35}$ etc. No numerical study of PDL damage has considered softening effects due to fibre alignment or fibrous network failure. Furthermore, the damage phenomena have not yet been implemented in a full FE model of PDL, considering its non-uniform thickness and porous fibrous structure.

The main objective of this study was to analyse the mechanical response of the PDL under traumatic conditions considering the softening phenomena caused by fibre alignment and rupture. A constitutive damage model was developed and incorporated into our previous material model. ${ }^{24}$ This damage model of PDL tissue was then implemented into an FE model of a human periodontium obtained by microCT. This FE model was subjected to three theoretical traumatic loads along intrusive, mesial-distal and labial-lingual directions.

This paper is organized as follows. First, we introduce the material model used to mimic the porous fibrous response of the PDL without damage. Then, the softening effect due to fibrous network alignment is formulated according to Mullins effect theory. The mechanical response caused by fibrous network failure is described and incorporated into the constitutive model. Then, the parameters of the material model are obtained by an iterative process until good fitting of experimental data from the literature is achieved. The porous fibrous hyperelastic damage material model is implemented in a user material subroutine in Abaqus (Abaqus 
6.14, Simulia, Rhode Island, USA) and applied to PDL tissue in a microCT 3D FE model. Finally, the results of fibrous alignment and fibrous failure and their effects on PDL behaviour under traumatic loading are extensively discussed.

\section{2 | MATERIAL AND METHODS}

\subsection{Material model characterization}

In this section, first, the non-damage material model, which takes into account the different behaviours under tensile and compressive forces, is explained. Second, the formulation of the softening phenomenon due to fibre alignment is presented, and the characterization process is explained. Finally, the damage mechanism due to fibre rupture is developed.

\subsubsection{Porous fibrous material model without damage}

It is well known that the PDL undergoes large deformation when the tooth is loaded. Thus, hyperelastic material models are useful to simulate large non-linear strains under constant strain rates. However, the PDL is a porous tissue filled with interstitial fluid, which causes a viscoelastic response, especially under compressive forces. To consider this effect, viscoelastic or porous-elastic material models should be used. Moreover, the collagen fibre network produces a directional dependence behaviour when the fibres are stretched. Consequently, a transversely isotropic hyperelastic material model should be used to simulate the fibrous behaviour of the ligament.

Due to these porous fibrous effects, we proposed in a previous paper ${ }^{24}$ a material model that considered both contributions. This material model combines the transversely isotropic hyperelastic behaviour caused by the collagen network and the time-dependent response produced by the interstitial fluid. However, it is not possible to correctly simulate the PDL response using a porous transversely isotropic hyperelastic material model for all loading conditions. Thus, we proposed a material model with different behaviours depending on the stretch direction. The material behaviour was defined by a porous transversely isotropic hyperelastic model or by a porous Ogden hyperfoam model if the region of the PDL was under tensile or compressive forces, respectively. The Ogden hyperfoam material model was developed for describing the behaviour of highly compressible elastomers. ${ }^{36}$ It was used by Bergomi et al. ${ }^{14}$ to describe the behaviour of the PDL according to the high compressibility (a Poisson ratio of 0.086 ) observed in their previous experimental study. ${ }^{6}$ The energy density function $(\psi)$ of the constitutive material model can be defined by the deviatoric $\left(\psi_{\text {dev }}\right)$ and volumetric $\left(\psi_{v o l}\right)$ components as follows:

$$
\psi(\widetilde{\boldsymbol{C}}, \mathbf{M})=\psi_{\text {dev }}\left(\widetilde{I_{1}}, \widetilde{I_{4}}\right)+\psi_{v o l}\left(J_{e l}\right) ; \quad \psi_{\text {dev }}\left(\widetilde{I_{1}}, \widetilde{I_{4}}\right)=\psi_{m}\left(\widetilde{I_{1}}\right)+\psi_{f}\left(\widetilde{I_{4}}\right)
$$

where $\psi_{m}$ is the component of the hyperelastic matrix, and $\psi_{f}$ is the component related to the fibrous network. Equation 1 is written in terms of the modified invariants $\widetilde{I_{1}}$ and $\widetilde{I_{4}}$, which are dilatational and deviatoric responses, respectively. These invariants are defined as follows:

$$
\tilde{I}_{1}=\operatorname{tr} \widetilde{\boldsymbol{C}} \quad \tilde{I}_{4}=\mathbf{m} \cdot \widetilde{\boldsymbol{C}} \cdot \mathbf{m}
$$


where $\mathbf{m}$ is a unitary vector defining the orientation of the collagen fibres in the reference configuration, and $\widetilde{\boldsymbol{C}}$ is the modified right Cauchy-Green tensor, defined by the modified deformation gradient $\widetilde{\boldsymbol{F}}$ as $\widetilde{\boldsymbol{C}}=\widetilde{\boldsymbol{F}}^{T} \widetilde{\boldsymbol{F}}$. The modified deformation gradient can be expressed as $\widetilde{\boldsymbol{F}}=J_{e l}-\frac{1}{3} \boldsymbol{F}$ where $J_{e l}$ is the Jacobian of the deformation gradient, and $\boldsymbol{F}$ is the associated deformation gradient defined by $\boldsymbol{F}=\frac{\partial \boldsymbol{x}}{\partial \boldsymbol{X}}$ where $\boldsymbol{X}$ and $\boldsymbol{x}$ define the respective position of a particle in the reference $\Omega_{0}$ and current $\Omega$ configurations. The terms of the energy density function can be written depending on $\tilde{I}_{4}$ as:

$$
\begin{aligned}
& \Psi_{m}=\left\{\begin{array}{c}
\frac{2 \mu}{\alpha^{2}}\left[\hat{\lambda}_{1}^{\alpha}+\hat{\lambda}_{2}^{\alpha}+\hat{\lambda}_{3}^{\alpha}\right] \quad \text { if }\left(\widetilde{I_{4}}<1\right) \\
C_{1} \cdot\left(\tilde{I}_{1}-3\right) \quad \text { if }\left(\widetilde{I}_{4} \geq 1\right)
\end{array}\right. \\
& \psi_{f}=\left\{\begin{array}{c}
0 \quad \text { if }\left(\widetilde{I}_{4}<1\right) \\
\frac{k_{1}}{2 \cdot k_{2}}\left\{\exp \left[k_{2} \cdot\left(\tilde{I}_{4}-1\right)^{2}\right]-1\right\} \quad \text { if }\left(\widetilde{I}_{4} \geq 1\right)
\end{array}\right. \\
& \psi_{v o l}=\left\{\begin{array}{c}
\frac{1}{\beta}\left[\left(J_{e l}\right)^{-\alpha \beta}-1\right] \quad \text { if }\left(\widetilde{I}_{4}<1\right) \\
\frac{1}{D}\left[\frac{\left(J_{e l}\right)^{2}-1}{2}-\ln J_{e l}\right] \text { if }\left(\widetilde{I}_{4} \geq 1\right)
\end{array}\right.
\end{aligned}
$$

In Equation 3, $\mu$ and $\alpha$ are material parameters for a hyperfoam material model. The stretch $\left(\hat{\lambda}_{i}\right)$ is defined as the ratio between the lengths at the deformed $(\boldsymbol{X})$ and reference configurations $(\boldsymbol{x})$ in the $i$ direction. In Equation $4, C_{1}$ is a material constant related to the ground substance of the transversely isotropic material model. In Equation 5, the fibrous term is 0 when the tissue is compressed. If not (Equation 6), $k_{1}>0$ and $k_{2}>0$ are the parameters that describe the exponential behaviour due to the presence of the collagen fibres. For the volumetric part of the strain energy function $\left(\Psi_{v o l}\right)$, in Equation $7, \beta$ determines the degree of porous hyperfoam material compressibility related to Poisson's ratio, by $\beta=v /(1-2 v)$, and $\alpha$ is the aforementioned material parameter in Equation 3. Finally, in Equation 8, $D$ is related to the bulk modulus, $K$, by $K=2 / D$.

The biphasic behaviour of the PDL was introduced by adding the porous contribution. Thus, the total stress in a point, $\sigma$, in the fully saturated tissue is defined ${ }^{37}$ as:

$$
\boldsymbol{\sigma}=(1-e) \cdot \overline{\boldsymbol{\sigma}}-e \cdot \bar{p}_{t} \cdot \boldsymbol{I}-\zeta \cdot \bar{p}_{t} \cdot \boldsymbol{I}
$$

where $\zeta$ is a factor that depends on saturation (in this case, $\zeta=1.0$ because the PDL is a fully saturated tissue), $e$ is the void ratio defined as the ratio of the volume of fluid $\left(V_{f}\right)$ to the total volume $\left(V_{t}\right)$ by $e=d V_{f} / d V_{t}, \bar{\sigma}$ is the effective stress of the solid matrix obtained from the second Piola-Kiorchhoff stress tensor of the strain energy function of the solid phase, and $\bar{p}_{t}$ is the average pressure stress of the fluid. The pressure stress, $\overline{p_{t}}$, is related to the Jacobian contribution from the permeability of the tissue by the non-linear Forchheimer flow law. It was employed in Abaqus (Abaqus 6.14, Simulia, Rhode Island, USA) to describe the fluid flow for permeability. The exponential permeability function described by Argoubi and Shirazi-Adl ${ }^{38}$ for biphasic materials was used to relate the fluid flow permeability dependence with the deformation as follows: 


$$
\bar{k}=k_{0}\left[\frac{e\left(1+e_{0}\right)}{e_{0}(1+e)}\right]^{2} \exp \left[M\left(\frac{1+e}{1+e_{0}}-1\right)\right]
$$

where $k_{0}$ and $e_{0}$ are the permeability and void ratio at zero strain, and $M$ is a dimensionless material parameter.

To fit the material parameters that describe the tensile and compressive response, two iterative processes were executed by a script written in Python ("Python 3.5.2, Python Software Foundation"). The material behaviour under tensile force was characterized by the experimental data obtained by Natali et al. ${ }^{28}$ In this experiment, the specimen was extracted from the mandible of an adult pig. It was cut into a rectangular shape containing a portion of both bone and tooth, with the PDL between the bone and tooth (schematically shown in Figure $3 \mathrm{~A}$ ), and its geometry was measured by a reflecting microscope. Then, the sample was stretched along the fibre direction until rupture using a material testing machine (Zwick ${ }^{\circledR}$ Z005/TN29). The compressive response was obtained by fitting experimental data obtained by Bergomi et al. ${ }^{14}$ using bovine samples. The experimental results were obtained by Bergomi et al. from a cylindrical specimen extracted from bovine first molar. The specimen was composed of a portion of a molar, bone and PDL (shown in Figure 3B). It was subjected to sinusoidal compressive displacement equivalent to $35 \%$ PDL thickness $(0.60 \mathrm{~mm})$ at three different frequencies $(0.1,0.5$ and $1 \mathrm{~Hz})$ using a displacement-controlled actuator (Microtester 5848, Instron, Norwood, MA, USA). Although porcine data are considered more optimal, ${ }^{17}$ some authors suggest there is not a marked difference in considering different species for compression loads. ${ }^{28}$ The similarity between the compressive response of different species will be verified later in section 3.1.1, comparing the response of the material model (characterized with bovine experimental data) with the compressive response of PDL samples from cats.

The material parameters were obtained in separate steps. First, $C_{1}$ was obtained by fitting the first region of the experimental curve ${ }^{28}$ using the neo-Hookean hyperelastic model response. Afterwards, $k_{2}$ and $k_{1}$ were determined by mimicking the curvature and fitting the rest of the experimental tensile curve. ${ }^{28}$ Figure $4 \mathrm{~A}$ shows the material response variation for different values of $C_{1}, k_{1}$ and $k_{2}$. The permeability $\left(k_{0}\right)$ was defined by fitting the experimental compressive behaviour at $1 \mathrm{~Hz}{ }^{14}$ using the proposed material model. To avoid unreal $k_{0}$ values, the permeability was constrained between $\left(10^{-15} \mathrm{~m}^{2}\right)$ and $\left(10^{-13} \mathrm{~m}^{2}\right)$ according to the permeability dimension order of other studies. ${ }^{14,39}$ The dependence of the compressive behaviour on the permeability value is shown in Figure 4C. The fitting procedures were widely explained in our previous work. ${ }^{24}$ Finally, the material model described was implemented in the UMAT user subroutine in Abaqus 6.14 commercial software. 


\subsubsection{Softening due to fibre alignment}

Several authors 19,29,40-42 experimentally observed a softening behaviour of the PDL during preconditioning loading-unloading cycles. This effect was shown in experimental studies both for bovine ${ }^{41,42}$ and porcine samples. ${ }^{29,40}$ This stress softening phenomenon is commonly referred to as the Mullins effect. ${ }^{43}$ Although this effect was formulated to represent the preconditioning response in synthetic rubbers, it has been extensively used for soft tissues. ${ }^{44}$ Microscopically, the softening phenomenon allows for multiple interpretations, such as fibre slipping, fibrous network reorganization or fibre separation from the ground substance. Some of these softening mechanisms are illustrated in Figures 2A, 2B and 2C.

To represent the softening phenomenon, an anisotropic extension of the pseudo-elastic model proposed by Ogden and Roxburgh ${ }^{45}$ and Horgan et al. ${ }^{46}$ was used. The energy density function defined in Equation 1 by the deviatoric and volumetric components was modified in the following form:

$$
\psi\left(\widetilde{I_{1}}, \widetilde{I_{4}}, \eta\right)=\eta \psi_{\text {dev }}\left(\widetilde{I_{1}}, \widetilde{I_{4}}\right)+\Phi(\eta)+\psi_{v o l}\left(J_{e l}\right)
$$

where $\Phi(\eta)$ and $\eta$ are the damage continuous function and the damage variable, respectively. The continuous function $\Phi$ is defined by the expression of the Ogden-Roxburgh model as follows:

$$
\Phi(\eta)=\frac{\left(m+\beta_{m} \psi_{d e v}^{m}\right)}{r \sqrt{\pi}}\left\{\exp \left[-\left(\frac{\psi_{d e v}^{m}-\psi_{d e v}}{m+\beta_{m} \psi_{d e v}^{m}}\right)\right]^{2}-1\right\}+(1-\eta) \psi_{d e v}^{m}
$$

where $r$ and $\beta_{m}$ are dimensionless material parameters, $m$ has the dimensions of energy, and $\psi_{d e v}^{m}$ is the maximum value of the deviatoric strain energy density experienced by the material during its deformation. The damage variable $\eta$ varies with the deformation according to:

$$
\eta=1-\frac{1}{r} \operatorname{errf}\left(\frac{\Psi_{d e v}^{m}-\psi_{d e v}}{m+\beta_{m} \psi_{d e v}^{m}}\right)
$$

where the error function $(\operatorname{err} f)$ is defined as $\operatorname{err} f(x)=\frac{2}{\sqrt{\pi}} \int_{0}^{x} \exp \left(-\omega^{2}\right) d \omega$.

To obtain the different parameters of the Mullins effect damage model, a non-linear regression procedure was implemented in MATLAB (MATLAB 6.0 R12 The MathWorks Inc., Natick, MA, 2000). Although the experimental data of Natali et al. ${ }^{28}$ was used in the previous section, the experimental data of one of their previous studies ${ }^{29}$ was used for the characterization of this softening phenomenon. Although the same animal species was used for both tests, there are significant differences in the results of both studies. These differences could be due to the fact that different regions of the PDL were tested or that different strain rates were imposed. Nevertheless, it was assumed that Natali et al. utilized similar experimental protocols in both studies, obtaining a similar softening response with the preconditioning cycles. According to the test data of Natali et al, ${ }^{29}$ the preconditioning of the tissue was defined as a change in the hysteresis curve until a steady condition was reached. The hysteresis indicates an energy loss within the PDL between cycles that is defined in this manuscript as a softening phenomenon caused by the fibrous network reorganization. Therefore, the softening phenomenon was characterized by preconditioning the experimental 
data from Natali et al. ${ }^{29}$ The extraction protocol and the geometric registration procedure followed by Natali et al. ${ }^{29}$ was the same as that explained in section 2.1.1 for the experimental test described in the previous experimental study by Natali et al. ${ }^{28}$ However, the specimen of this study was subjected to loading and unloading cycles of $40 \%$ PDL thickness at a constant elongation rate of $0.5 \mathrm{~mm} / \mathrm{s}$. The parameters obtained $\left(r, \beta_{m}\right.$ and $\left.m\right)$ were then implemented in our material model. As described previously, the behaviour of our material model was characterized by the data in Natali et al. ${ }^{28}$ The softening phenomenon was then added to the aforementioned user subroutine in Abaqus. The complete strain energy function is detailed in Equation 20.

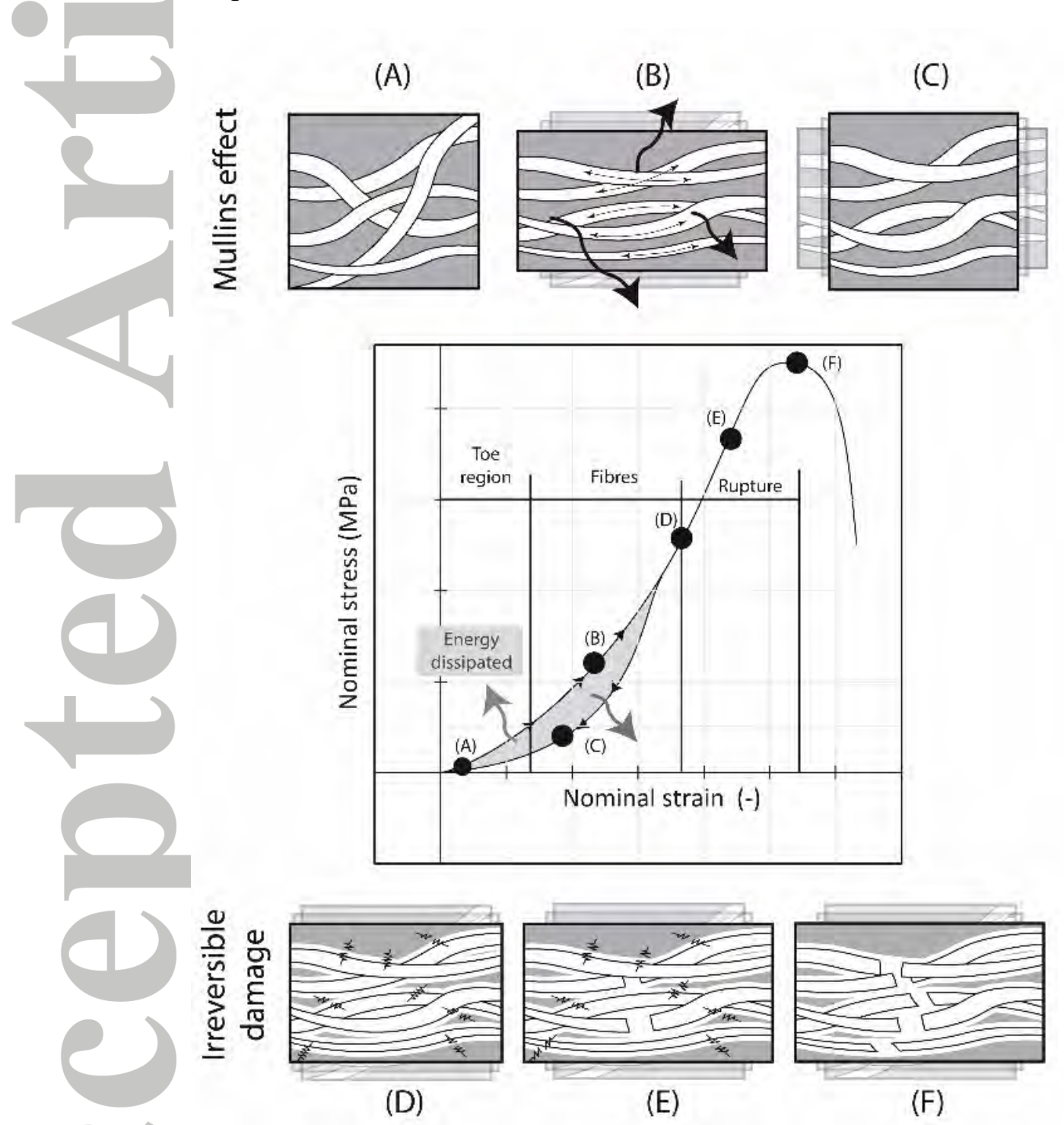

FIGURE 2 Schematic of the PDL tensile response: (A) Disorganized fibrous network at the initial state, (B) Fibre alignment and energy dissipation due to the friction between fibres during tensile loading, (C) Organized fibrous network after load removal, (D) Damage to the ties between the fibres and the matrix beginning at higher strains, (E) Fibre rupture at strains higher than the fibre elongation, (F) Rupture of the entire fibrous network and tissue failure. 


\subsubsection{Damage due to fibre over-elongation}

In addition to the softening phenomenon due to loading-unloading cycles, PDL damage can be caused by the rupture of collagen fibres or damage to the fibre attachments to the ground matrix. ${ }^{47}$ Under physiological loads, the waviness of the fibres makes the tissue softer for low strains (see the region in Figure 2) and stiffer for high strains. However, the damage phenomenon starts when the strain exceeds a specific limit (Figure 2D). This damage leads to a decrease in tissue stiffness. Any further increase in loading (Figure 2E) leads to progressive failure of the collagen fibre network until its complete rupture (Figure 2F).

Several authors have used FE models to determine the PDL stress state. ${ }^{23,48,49}$ However, few of them ${ }^{16,28}$ described the PDL behaviour in the failure region. As previously mentioned, the study of Natali et al. ${ }^{28}$ is one of the most relevant. Nevertheless, their study did not consider softening behaviour due to fibre alignment, the pressure of the interstitial fluid or its effect on a full PDL. To include these aspects, a porous transversely isotropic hyperelastic damage constitutive model is developed in the present work. Thus, continuum damage mechanic was used to describe the irreversible damage under finite deformation.

To establish the law of evolution, the equivalent strain ${ }^{50}$ of the fibrous term $\boldsymbol{\Xi}_{\boldsymbol{s}}$ at any time s of the loading process is defined as:

$$
\Xi_{s}(\widetilde{\boldsymbol{C}})=\sqrt{2 \cdot \psi_{f}\left(\widetilde{I_{4}}\right) \cdot \widetilde{\boldsymbol{C}}(s)}
$$

On the other hand, $\Xi_{t}^{m}$ is the maximum value of $\Xi_{s}$ over the past history up to the current time s, which can be mathematically expressed as:

$$
\Xi_{t}^{m}=\max _{s \in(0, t)}\left\{\sqrt{2 \cdot \psi_{f}\left(\widetilde{I_{4}}\right) \cdot \widetilde{\boldsymbol{C}}(s)}\right\}
$$

where $t$ is the time when the damage starts. With $\Xi_{s}$ and $\Xi_{t}^{\max }$ defined, the damage criterion in the strain space can be expressed for any time $t$ when the following expression is fulfilled:

$$
\begin{array}{l|l}
\Xi_{s}-\Xi_{t}^{m} \leq 0 & (16) \\
\hline
\end{array}
$$

Equation 16 represents the damage surface in the strain space whose evolution is determined by the rate of the damage parameter $D_{f}\left(\Xi_{t}^{m}\right) \in[0,1]$ :

$$
\frac{d D_{f}}{d t}=\left\{\begin{array}{cl}
0 & \text { if } \Xi_{s}<\Xi_{t}^{m} \\
\bar{h}\left(\Xi_{t}^{m}\right) \cdot \Xi_{t}^{m} & \text { if } \Xi_{s} \geq \Xi_{t}^{m}
\end{array}\right.
$$

where $\bar{h}\left(\Xi_{t}^{m}\right)$ is the function that characterizes the damage process of the fibrous network. In this model, we adopted the damage function used by Rodriguez et al. ${ }^{51}$, as follows:

$$
\bar{h}\left(\Xi_{t}^{m}\right)=\frac{\alpha_{f}}{\beta_{f}} \frac{\left(4 \Xi_{t}^{m} \alpha_{f}+\beta_{f}\right) e^{2 \alpha_{f}\left[\left(2 \Xi_{t}^{m} / \beta_{f}\right)-1\right]}}{\left\{1+\alpha_{f} \Xi_{t}^{m} e^{2 \alpha_{f}\left[\left(2 \Xi_{t}^{m} / \beta_{f}\right)-1\right]}\right\}^{2}}
$$

After integration of Equation 17, the damage parameter $\left(D_{f}\right)$ can be expressed as a function of two material parameters, $\alpha_{f}$ and $\beta_{f}$, as follows: 


$$
D_{f}\left(\Xi_{t}^{m}\right)=\frac{1}{2}\left\{1+\frac{\alpha_{f} \Xi_{t}^{m} e^{2 \alpha_{f}\left[\left(2 \Xi_{t}^{m} / \beta_{f}\right)-1\right]}-1}{\alpha_{f} \Xi_{t}^{m} e^{2 \alpha_{f}\left[\left(2 \Xi_{t}^{m} / \beta_{f}\right)-1\right]}+1}\right\}
$$

This damage function allows control of the exponential damage at the beginning, the close constant damage propagation and the end of damage. The $\left(1-D_{f}\right)$ term multiplies the fibrous term of the deviatoric component (Equation 20). Thus, the porous transversely isotropic hyperelastic damage constitutive model can be summarized as:

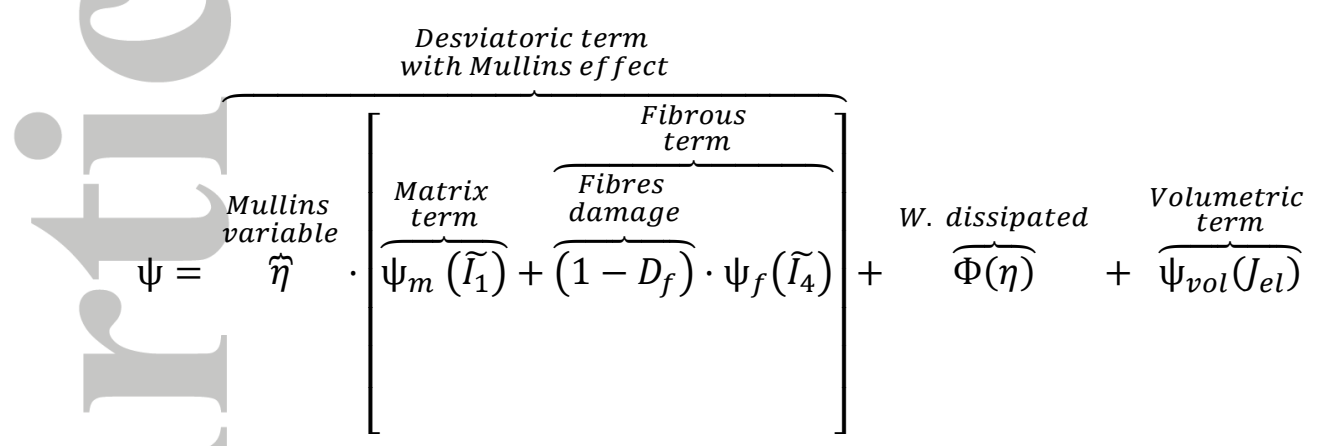

To obtain the parameters that describe the material model damage, the constitutive formulation presented (Equation 20) was implemented in an UMAT user subroutine. The scheme of the FE program used to implement the porous fibrous hyperelastic damage material model is described in Appendix 2. An iterative process was used to fit the tensile test results obtained by Natali et al. ${ }^{28}$ The iterative process was executed in Python 3.5.2 and was stopped when the deviation between the numerical and experimental curves was lower than $2 \%$. It was considered that the tissue damage starts at a strain value of 0.6 (corresponding to an equivalent strain value, $\Xi_{t}$, of 0.28 ).

\section{2 | FE simulations of the test specimens}

In this section, the material model described previously was implemented using a FE analysis to mimic the experimental test performed by Natali et al. under tensile forces. ${ }^{28}$ Moreover, the experimental tests performed by Bergomi et al. ${ }^{14}$ and Nishihira et al. ${ }^{52}$ were simulated to analyse the material response under compressive forces. 


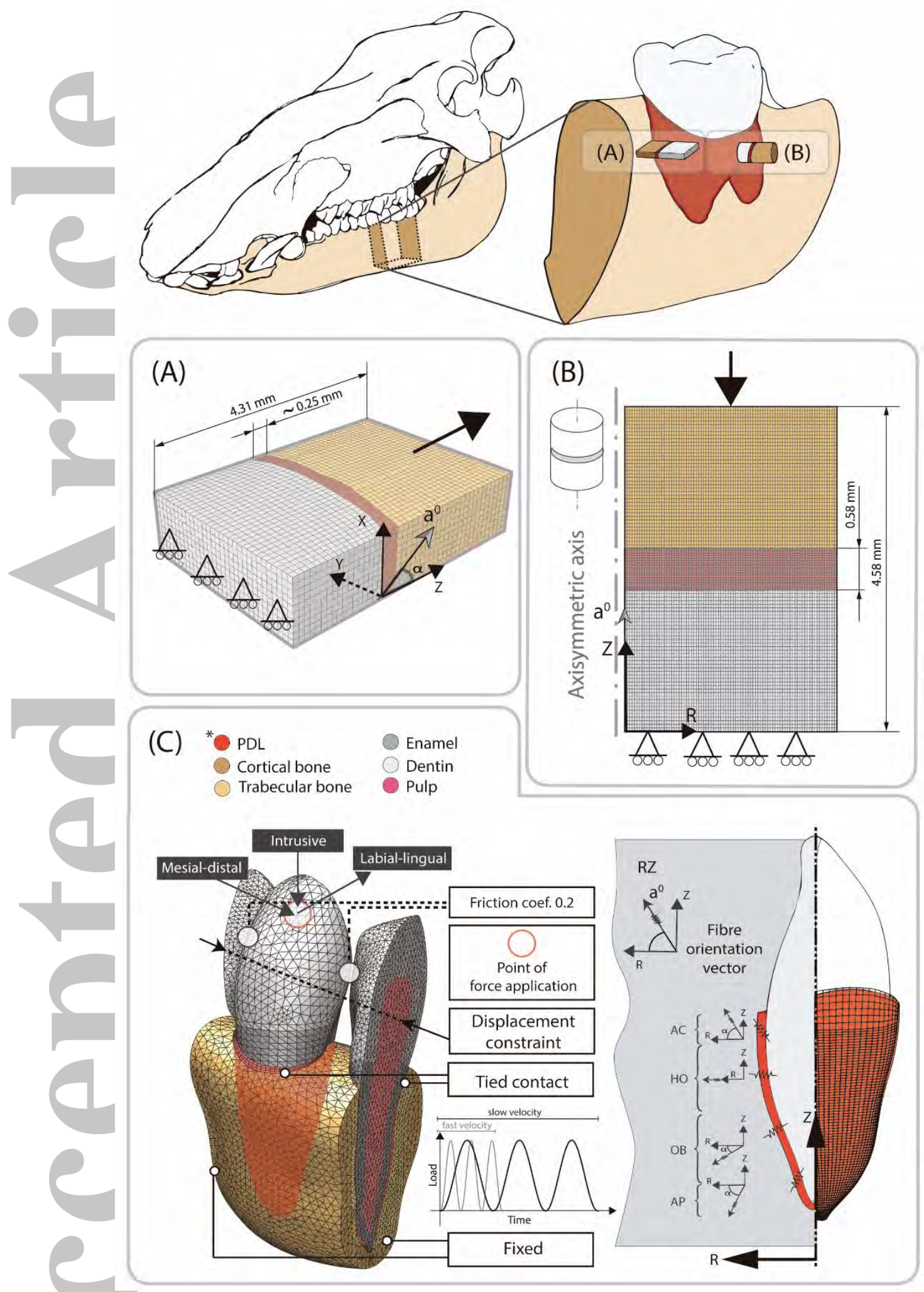

FIGURE 3 Top: Schematic of the acquisition of the experimental samples by Natali et al. ${ }^{16}$ and Bergomi et al. ${ }^{14}$ (A) A 3D FE model that mimics the sample used by Natali et al. ${ }^{16}$ in their experimental test and boundary conditions applied. (B) An axisymmetric FE model that mimics the samples used by Bergomi et al. ${ }^{15}$ in their experiments and the boundary conditions applied. (C) Left: The FE model based on a microCT scan with the boundary conditions and loading cycles. Right: Mesh of the canine PDL and fibre distribution according to the different directions of fibre bundles (AP: apical, OB: oblique, HO: horizontal and AC: alveolar crest). *For colour interpretation, see the web version of this article. 
A 3D FE model reproducing the test sample geometry used experimentally by Natali et al. ${ }^{16}$ was developed using Abaqus CAE 6.14 (Abaqus 6.14, Simulia, Rhode Island, USA). Development of the FE model (Figure $3 \mathrm{~A}$ ) was extensively explained in our previous work. ${ }^{24}$ After a mesh refinement process, the average size of the elements was approximately 0.035 $\mathrm{mm}$. The material parameters of the transversely isotropic hyperelastic contribution (Equations 4 and 6) were obtained by mimicking the experimental dataset reported by Natali et al. ${ }^{28}$ The specimen was extracted from the mandible of an adult pig. It was cut into a rectangular shape containing a portion of both bone and tooth with the PDL between the bone and tooth (shown schematically in Figure 3a), and its geometry was measured by a reflecting microscope. Then, the sample was stretched along the fibre direction until rupture using a material testing machine (Zwick ${ }^{\circledR}$ Z005/ TN29). Therefore, the characterization process presented in section 2.1.1 considers that PDL fibres run in the direction of the load (90 degrees). To mimic the specific clamping fixture used in the experimental test, the bottom surface of the tooth was fixed, and a displacement equivalent to $70 \%$ PDL thickness $(0.25$ $\mathrm{mm}$ ) was applied at the top surface of the bone. The displacement was computed from the experimental data reported by Natali et al. ${ }^{28}$ It was assumed that the stress-stretch curve data were only caused by the PDL stretch because bone and teeth were considered practically rigid. ${ }^{14,28}$ Furthermore, the response of the test sample was evaluated for different fibre orientations $\left(90^{\circ}, 70^{\circ}, 45^{\circ}, 20^{\circ}\right.$ and $\left.0^{\circ}\right)$ (Figure $3 \mathrm{~A}$ ). The material parameters for the different FE model regions appear in Table 1.

For softening phenomenon verification during the tensile preconditioning cycles, the 3D FE model based on the dataset of Natali et al was used. ${ }^{16}$ The experiment included six pure sinusoidal traction displacements equivalent to $20 \%, 24 \%, 28 \%, 32 \%, 36 \%$ and $40 \%$ PDL thickness $(0.25 \mathrm{~mm})$ at $1.0 \mathrm{~Hz}$ (Figure $5 \mathrm{~A})$. These strain values correspond to the nondamaged strain region of the fibrous network, according to the experimental results of Natali et al. ${ }^{28}$ ( higher strain than $60 \%$ of the PDL thickness). Moreover, the dependency of the softening effect on the fibre direction was analysed. Thus, several simulations for different $\alpha$ values in the undeformed state $\left(0^{\circ}, 20^{\circ}, 45^{\circ}, 70^{\circ}\right.$ and $90^{\circ}$ ) (Figure $5 \mathrm{C}$ ) were performed.

On the other hand, the 3D FE model was subjected to a displacement equivalent to $135 \%$ of the PDL thickness to determine the material parameters that define fibrous network damage. According to Natali's experimental results, ${ }^{28}$ the test sample failed before this strain value. The material damage parameters were obtained by an iterative process executed by a script written in Python until good agreement with the experimental results was achieved.

For characterization of the compressive material parameters, the axisymmetric FE model (Figure 3B) developed by Bergomi et al. ${ }^{14}$ was used. The response of the tissue without softening phenomena was evaluated at $0.1 \mathrm{~Hz}, 0.5 \mathrm{~Hz}$ and $1 \mathrm{~Hz}$ frequencies (Figure 4B).

To verify the softening phenomenon due to fibrous alignment, the axisymmetric FE model was subjected to three compressive cycles equivalent to $15 \%, 25 \%$ and $30 \%$ of the PDL thickness $(0.58 \mathrm{~mm})$. Moreover, to understand the dependency of this effect on the strain rate, a previous test was performed at $0.1 \mathrm{~Hz}, 0.5 \mathrm{~Hz}$ and $1.0 \mathrm{~Hz}$ frequencies. 
As mentioned above, few differences exist between the compressive behaviour of PDL from different species. This fact was verified by numerically mimicking the experimental data of adult cat samples ${ }^{52}$ with a material model based on bovine experimental data. Thus, the 3D FE model of Natali et al. ${ }^{28}$ was compressed at the same strain rates of $260 \%, 63 \%, 6.7 \%$, $0.55 \%$ and $0.0052 \%$ of PDL thickness per second, as used by Nishihira et al. ${ }^{52}$, and the results were compared.

\section{3 | PDL material model application to a canine microCT FE model}

After characterization and validation of the porous transversely isotropic hyperelastic damage material model, this material model was implemented for the PDL tissue in an FE model of a portion of a human mandible obtained by microCT. In this section, the FE model and the boundary conditions are explained.

A portion of a human mandible was extracted from a cadaver, and it was conserved under stable conditions until it was scanned. The tomograms obtained from the scanning were rebuilt, the noise was reduced, and the point cloud was converted into Non-Uniform Rational Bases Splines (NURBS). This model was composed of a full canine tooth with its PDL, two portions of lateral teeth (premolar and lateral incisor) with their respective ligaments, and portions of cortical and trabecular bone of the mandible (shown in Figure 3C). In addition, the teeth were divided into pulp, enamel and cement regions (shown in the tooth section of Figure $3 \mathrm{C}$ ). The microCT FE model was meshed using a commercial software program (Abaqus 6.14, Simulia, Rhode Island, USA) until mesh convergence. More details of the model are provided in our previous paper. ${ }^{24}$ The material properties applied to each region of the model are summarized in Table 1 . The influence of the softening effects was analysed in the complete FE model.

Regarding the loading conditions, the FE model was subjected to three theoretical loads of $360 \mathrm{~N}$ along intrusive, labial-lingual and mesial-distal directions (see the load point application in Figure 3C). The intrusive load is a vertical load defined by the main longitudinal axis of the teeth and oriented along the direction from to the tooth cuspid to the tooth root. The magnitude of this load was defined according to different studies of adult human teeth under possible damage conditions. ${ }^{1,53,54}$. There are significant discrepancies in the literature regarding the magnitude of the physiological occlusal load, which can vary between $14.5 \mathrm{~N}^{55}$ to $100 \mathrm{~N}{ }^{1}$ In comparison with normal chewing conditions, a $360 \mathrm{~N}$ load would represent a large load that may cause damage to the ligament, which is demonstrated in the results section. However, this magnitude does not represent a real traumatic load of an occlusal trauma scenario. Loads were applied by three sinusoidal cycles (see the detail of Figure 3C) to analyse the damage due to load repetition. Moreover, each case was studied considering a physiological chewing velocity $(1.33 \mathrm{~Hz})^{56}$ and a theoretical slow velocity $(0.0166 \mathrm{~Hz})$. The results at fast (physiological) and slow velocities allow us to separately study the residual strains due to the porous coupling and the softening phenomena. The lateral nodes of the mandible were constrained considering fixation with the rest of the mandible. Moreover, the nodes of the cut planes of the premolar and incisal teeth were 
constrained at the mesial-distal direction to represent the restriction imposed by the remaining teeth. The contact between teeth was defined by a penalty contact term with a friction coefficient (value of 0.2 ), according to the studies by Zheng and Zhou. ${ }^{57}$ Figure $3 \mathrm{C}$ shows the boundary and loading conditions applied. On the other hand, the PDLs were considered to be attached to the trabecular bone and the teeth by a tied contact due to the presence of interstitial fibres. ${ }^{4,48,58}$

TABLE 1 Mechanical properties assigned to each region.

\begin{tabular}{|c|c|c|c|c|c|c|c|c|}
\hline & \multicolumn{4}{|c|}{ Ground parameters } & \multicolumn{4}{|c|}{ Porous properties } \\
\hline & \multicolumn{2}{|c|}{$\begin{array}{l}\text { Young's Modulus } \\
\qquad(\mathrm{MPa})\end{array}$} & \multicolumn{2}{|c|}{$\begin{array}{c}\text { Poisson's Ratio } \\
(-)\end{array}$} & $\begin{array}{c}k_{0} \\
\left(\mathrm{~m}^{2}\right)\end{array}$ & $\begin{array}{l}M \\
(-)\end{array}$ & $\begin{array}{l}\text { Void Ratio } \\
\qquad e_{0}(-)\end{array}$ & $\begin{array}{l}\text { Specif. weight of } \\
\text { interstitial fluid } \\
\left(\mathrm{N} / \mathrm{mm}^{3}\right)\end{array}$ \\
\hline Pulp & \multicolumn{2}{|c|}{$2^{a}$} & \multicolumn{2}{|c|}{$0.45^{b}$} & - & - & - & - \\
\hline Enamel & \multicolumn{2}{|c|}{$80000^{c}$} & \multicolumn{2}{|c|}{$0.31^{b}$} & - & - & - & - \\
\hline Cortical bone & \multicolumn{2}{|c|}{$20000^{e}$} & \multicolumn{2}{|c|}{$0.30^{e}$} & & & & \\
\hline Trabecular bone & \multicolumn{2}{|c|}{$345^{\mathrm{d}}$} & \multicolumn{2}{|c|}{$0.31^{d}$} & $5.29 \cdot 10^{-14 d}$ & - & $4^{d}$ & $9.8 \cdot 10^{-6}$ \\
\hline \multirow[t]{2}{*}{ Dentin } & \multicolumn{2}{|c|}{$15000^{d}$} & \multicolumn{2}{|c|}{$0.31^{\mathrm{d}}$} & $3.88 \cdot 10^{-1 / d}$ & - & $4^{\mathrm{d}}$ & $9.8 \cdot 10^{-6}$ \\
\hline & $\begin{array}{c}\mu \\
(\mathrm{MPa})\end{array}$ & $\begin{array}{l}\alpha \\
(-)\end{array}$ & \multicolumn{2}{|c|}{$\begin{array}{c}\text { Poisson's Ratio } \\
(-)\end{array}$} & $\begin{array}{c}k_{0} \\
\left(\mathrm{~m}^{2}\right)\end{array}$ & $\begin{array}{l}M \\
(-)\end{array}$ & $\begin{array}{c}\text { Void Ratio } \\
e_{0}(-)\end{array}$ & $\begin{array}{l}\text { Specif. weight of } \\
\text { interstitial fluid } \\
\left(\mathrm{N} / \mathrm{mm}^{3}\right)\end{array}$ \\
\hline \multirow[t]{2}{*}{$\mathrm{PDL}$ if $\left(\widetilde{\boldsymbol{I}_{4}}<1\right)$} & $0.03^{d}$ & $20.9^{d}$ & \multicolumn{2}{|c|}{$0.257^{d}$} & $8.81 \cdot 10^{-15 d}$ & $14.2^{d}$ & $2.33^{d}$ & $9.8 \cdot 10^{-6}$ \\
\hline & $\begin{array}{c}C_{1} \\
(\mathrm{MPa})\end{array}$ & $\begin{array}{c}D \\
\left(\mathrm{MPa}^{-1}\right)\end{array}$ & $\begin{array}{c}k_{1} \\
(\mathrm{MPa})\end{array}$ & $\begin{array}{l}k_{2} \\
(-)\end{array}$ & $\begin{array}{c}k_{0} \\
\left(\mathrm{~m}^{2}\right)\end{array}$ & $\begin{array}{l}M \\
(-)\end{array}$ & $\begin{array}{l}\text { Void Ratio } \\
\qquad e_{0}(-)\end{array}$ & $\begin{array}{l}\text { Specif. weight of } \\
\text { interstitial fluid } \\
\left(\mathrm{N} / \mathrm{mm}^{3}\right)\end{array}$ \\
\hline PDL if $\left(\widetilde{I_{4}} \geq 1\right)$ & $0.01^{\dagger}$ & $9.078^{f}$ & $0.298^{f}$ & $1.525^{f}$ & $6.5 \cdot 10^{-15 f}$ & $9.5^{f}$ & $2.33^{d}$ & $9.8 \cdot 10^{-6}$ \\
\hline
\end{tabular}

\begin{tabular}{|c|c|c|c|c|c|c|}
\hline Softening phenomena & & ue to fib & ment & & le to $f$ & gation \\
\hline & $r(-)$ & $m(\mathrm{~mJ})$ & $\beta_{m}(-)$ & $\alpha_{f}(-)$ & $\beta_{f}(-)$ & $\Xi_{t}^{\max }(-)$ \\
\hline PDL damage & 1.492 & 0.064 & 0.1 & 1.0 & 0.25 & 0.28 \\
\hline${ }^{\mathrm{a}}$ Lin et al. 2014 & & & & & & \\
\hline${ }^{\mathrm{b}}$ Belli et al. 2017 & & & & & & \\
\hline${ }^{c}$ Nikolaus et al. 2016 & & & & & & \\
\hline${ }^{\mathrm{d}}$ Bergomi et al. 2011 & & & & & & \\
\hline${ }^{\mathrm{e}}$ Lacroix and Prenderga & 2002 & & & & & \\
\hline${ }^{f}$ Ortún-Terrazas et al. 20 & & & & & & \\
\hline
\end{tabular}




\section{3 | RESULTS}

\section{1 | Material model characterization and validation}

This section shows the material parameter characterization and the numerical results for the mimicked experimental test (previously defined in section 2.2). First, the mechanical response of the material model without damage is presented. Then, the Mullins effect is evaluated in compression and tension tests, modifying the fibre bundle direction and the strain rate. Finally, the fibrous network damage is analysed using experimental data. ${ }^{28}$

\subsubsection{Porous fibrous material model without damage}

Figure 4 shows the responses of the material model without damage defined in section 2.1.1. The results of mimicking the experimental tests performed by Natali et al., ${ }^{28}$ Bergomi et al. ${ }^{14}$ and Nishihira et al. $^{52}$ are summarized in Figures $4 \mathrm{~A}, 4 \mathrm{~B}$ and $4 \mathrm{C}$, respectively.

Figure 4A shows a good approximation between the finite element analysis (FEA) at $90^{\circ}$ and the experimental responses under tensile efforts less than 0.7 strain. The higher deviation appears in the first part of the curve due to the concave shape of the Neo-Hookean material response. The dependence of the material behaviour response on the direction of the fibres is also shown (Figure 4A). The stiffness of the PDL increases when the fibre bundles run along the direction of the traction load. The values of $k_{1}$ and $k_{2}$ modify the curvature and exponential growth of the curve, respectively (as shown in Figure 4A). For a region in which fibres are aligned at $20^{\circ}$ or $0^{\circ}$, the material response is mainly defined by the porous hyperelastic behaviour of the matrix (Equation 4). Increasing the $C_{1}$ parameter leads to stiffer behaviour of the porous hyperelastic matrix (as shown in Figure 4A).

The energy dissipation due to the porous term is clearly presented in Figure 4B. Despite the difficulties in predicting the permeability effects, the FEA curves closely follow the experimental data of Bergomi et al. ${ }^{14}$ For higher strain rates, the fluid phase does not have enough time to flow, and consequently, the tissue becomes stiffer. An increase in the initial permeability value $k_{0}$ leads to softer behaviour of the tissue because the fluid can more easily flow out (as shown in Figure 4B).

Although different samples from species were used for tensile (porcine samples) and compressive (bovine samples) material behaviour characterization, it was demonstrated that the different species have similar compressive responses. Figure $4 \mathrm{C}$ compares the numerical results using our material model (characterized by data from bovine samples) and the experimental data of adult cat samples. ${ }^{52}$ It can be appreciated that no significant differences exist between the compressive responses of the PDL of each species. The similarity for high strain rates is especially remarkable when the fluid phase contributes more. 
(A) EX. Natali et al. 2008 -
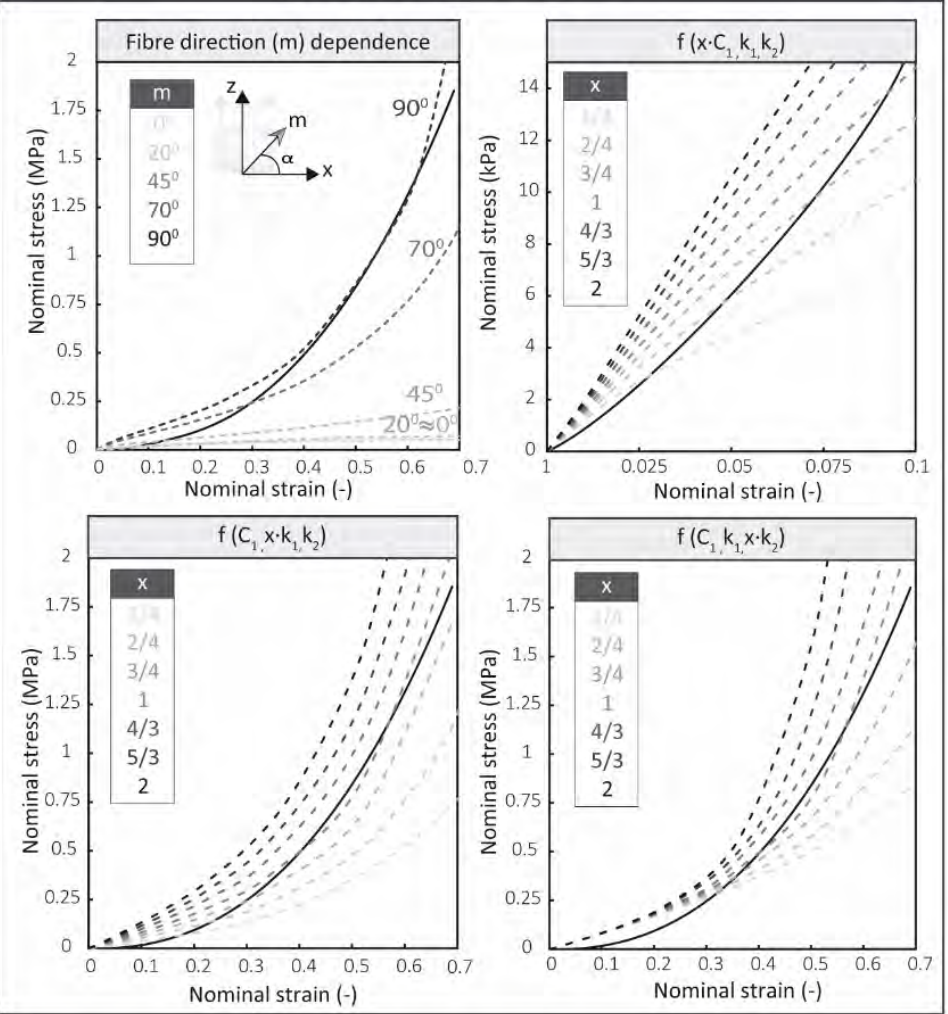

(B) EX Bergomi et al. 2011 $\triangle \square$
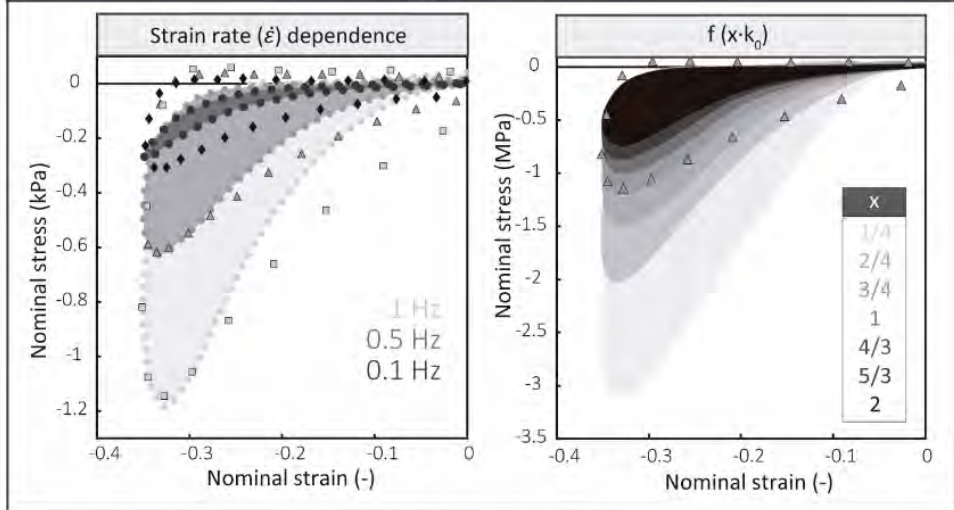

(C) Nishihira et al. $2003 \circ$

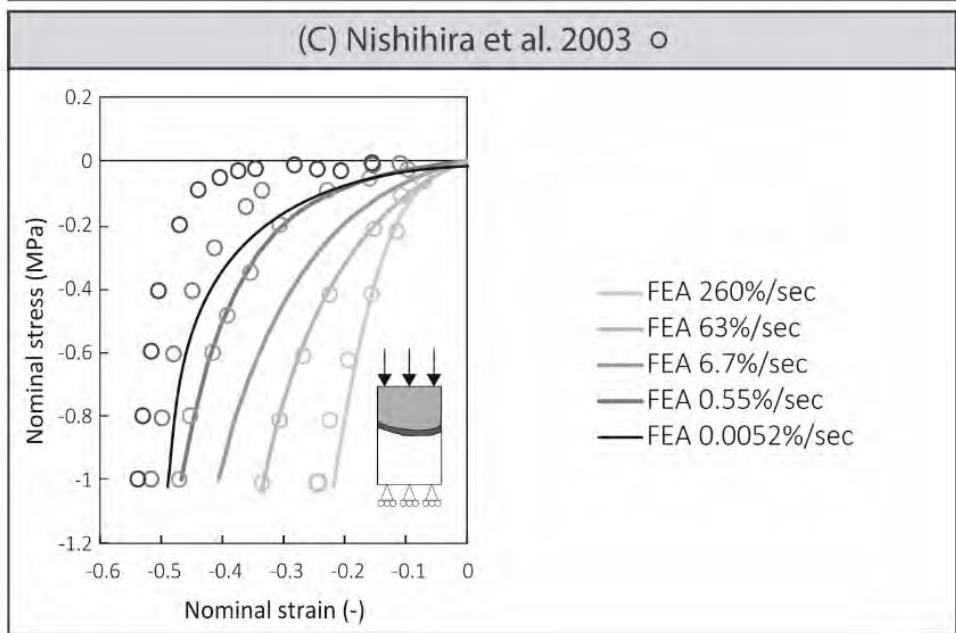

FIGURE 4 (A) Porous fibrous material model approximation to the experimental results of Natali et al. ${ }^{28}$ and its dependence on fibre direction and different parameter values $\left(C_{1}, k_{1}\right.$ and $k_{2}$ ). (B) Porous fibrous material model approximation to the experimental results of Bergomi et al. ${ }^{14}$ under $0.1 \mathrm{~Hz}, 0.5 \mathrm{~Hz}$ and $1 \mathrm{~Hz}$ frequencies and its behaviour dependence on different $k$ permeability values. (C) Comparison between the porous fibrous material model response and the experimental results obtained by Nishihira et al. $^{52}$ at $260 \% / \mathrm{sec}$, $63 \% / \mathrm{sec}, 6.7 \% / \mathrm{sec}, 0.55 \% / \mathrm{sec}$ and $0.0052 \% / \mathrm{sec}$ strain rates. The variation in the material parameters and permeability values was defined as the fraction $x$ of the values listed in Table 1. 


\subsubsection{Softening due to fibre alignment}

Figure 5 shows the FE analysis curves using the porous fibrous material model, which includes the softening behaviour caused by the fibre alignment (described in section 2.1.2) for different situations. Figure 5A shows the tensile response considering the Mullins effect characterized by the experimental data of Natali et al. ${ }^{29}$ By introducing the Mullins effect, the numerical response after the preconditioning cycles (Figure 5A) better fits the experimental data for the first part of the curve, where the porous fibrous material model without damage exhibited poor fitting. Modifying the $m$ and $r$ parameters leads to different energy loss and curvature, respectively, of the unloading curve (as shown in Figure 5B).

The dependence of the softening effect on the fibre bundle orientation and its behaviour under compressive effort is presented in Figures 5C and 5D, respectively. The Mullins effect at different fibre orientations is shown in Figure 5C. The effect of the fibre alignment is more noticeable when the fibres are aligned along the loading direction. Moreover, the porous and Mullins effects are coupled, as shown in the graphical detail of Figure 5C. In a pure Mullins effect response, the unloading and subsequent loading curves must be the same. However, a deviation occurs when the porous effect is added. This effect was also observed during compression, revealing a softening phenomenon when the loading process is repeated (Figure $5 \mathrm{C})$.

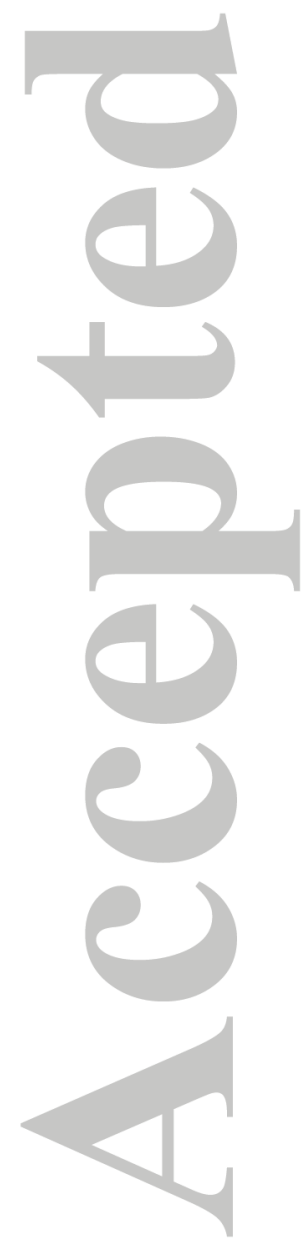



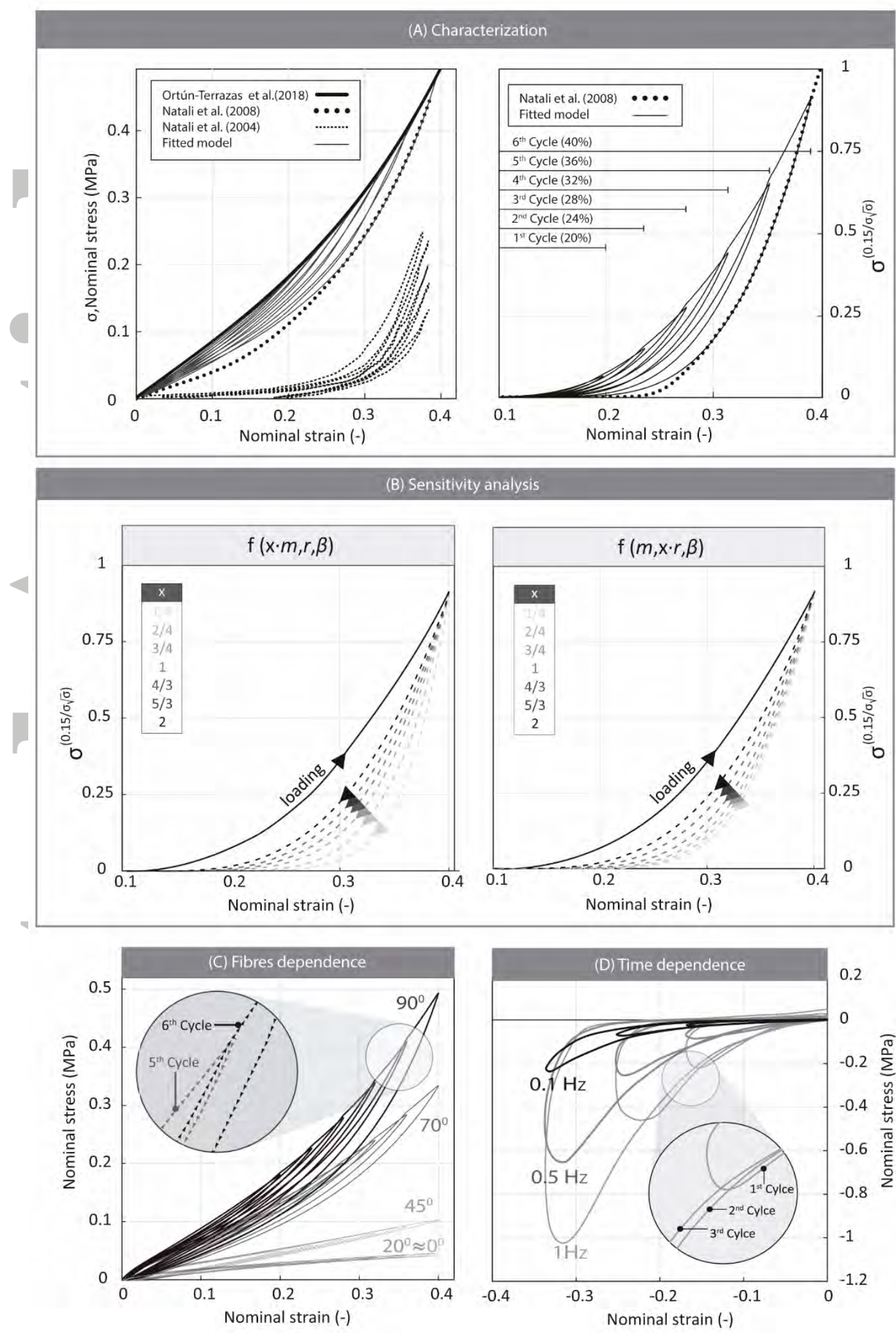

FIGURE 5 (A) Left: Comparison between Natali's two previous studies, ${ }^{28,29}$. Right: representation of the theoretical Mullins effect using a magnification variable of the nominal stress $\left(\sigma^{0.15 / \sigma \sqrt{\sigma}}\right)$. (B) Dependence of the softer unloading response with different $m$ and $r$ material parameters. (C) Mullins effect for different fibre directions using the 3D FE model of the Natali et al. ${ }^{16}$ experiment. (D) Mullins effect for different strain rates using the axisymmetric FE model of the Bergomi et al. ${ }^{14}$ experiment. 


\subsection{3 | Damage due to fibre over-elongation}

Figure 6 shows several aspects of the damage function (described in section 2.1.3). The dependence of the damage function on different damage parameters and the curve fitted to the experimental results of Natali et al. ${ }^{28}$ are shown in Figure $6 \mathrm{~B}$. Figure $6 \mathrm{C}$ shows the damage evolution of the elements of the PDL mesh.

Varying the parameters $\alpha_{f}$ and $\beta_{f}$ can increase the damage initiation velocity, the gradient of damage evolution in the quasilinear zone and the final failure evolution (Figure 6A). Moreover, extension of the damage region is a function of these parameters. The PDL begins to become damaged at strains higher than 0.6 ( 0.28 equivalent strain of the fibrous term), and at a strain of 1.05 , the tissue has completely failed. Therefore, PDL rupture occurs in a small strain range. Thus, similar parameters cause significant differences in tissue damage behaviour, as shown in Figure 6B. As mentioned, an iterative process was executed in Python to fit the damage parameters. For the iterative analysis, the 3D FE model mimicked the geometry of the sample used in Natali et al. ${ }^{16}$ The best adjustment was obtained for $\alpha_{f}=1$ and $\beta_{f}=0.25$ damage parameters values. The deviation between the experimental curve and the computational material response was lower than $2 \%$. Damage to the fibrous network starts to appear in two regions near the midplane (Figure $6 \mathrm{C}$, time $=3 \mathrm{~s}$ ), which are the thinnest regions, and continues to the closest regions until its complete failure (Figure 6C, time $=6 \mathrm{~s})$.

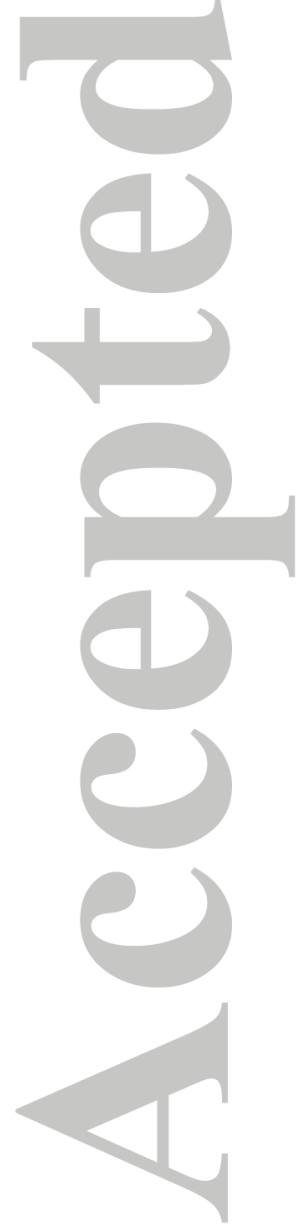




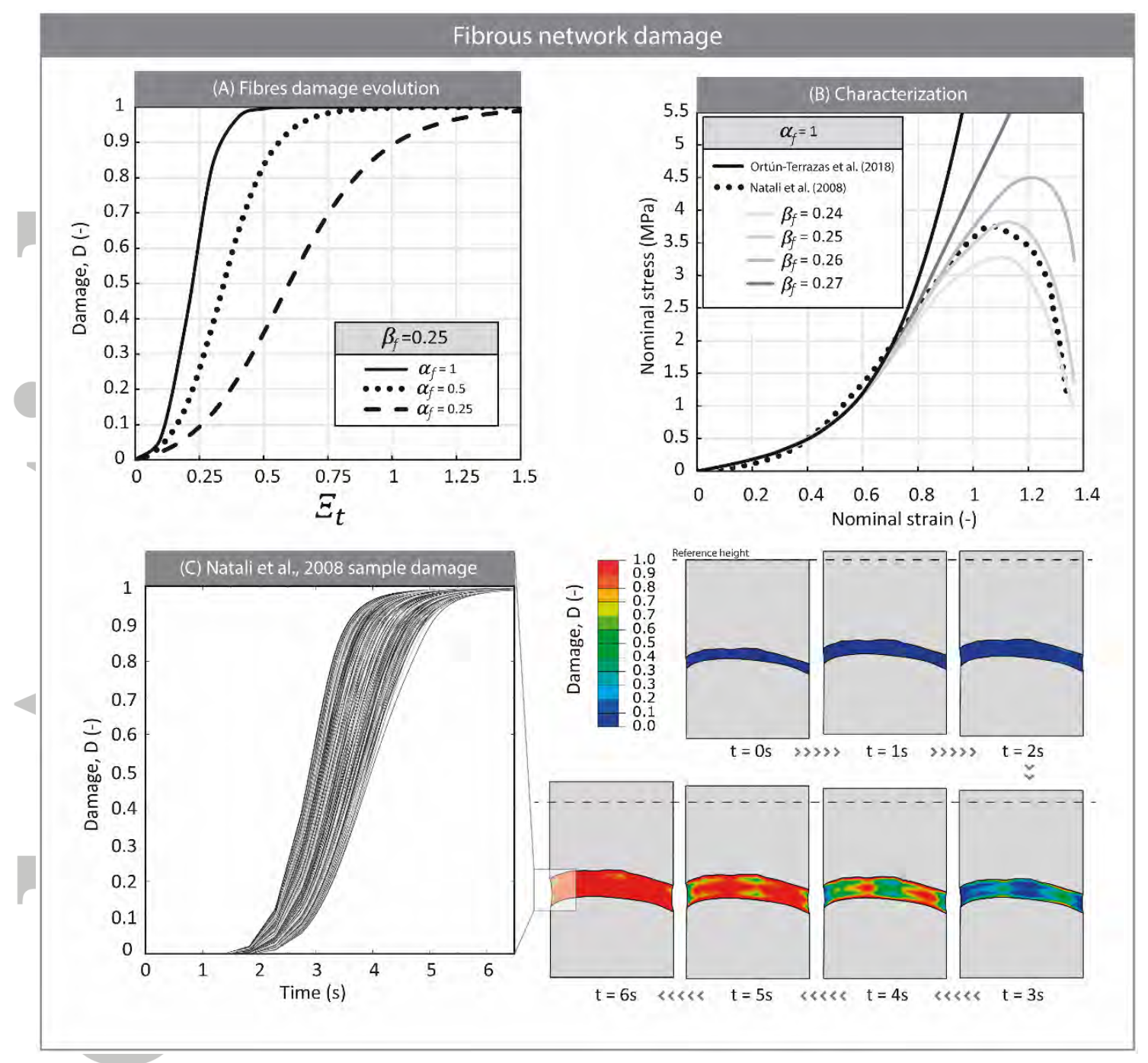

FIGURE 6 (A) Evolution of the damage function (Equation 19) modifying $\alpha_{f}$ and $\beta_{f}$ parameters. (B) Influence of $\alpha_{f}$ and $\beta_{f}$ damage parameters on the material model response and characterization of the fibrous network damage using the 3D FE model of the experimental dataset in Natali et al. ${ }^{16}$ (C) Left: damage evolution of the integration points of the 3D FE sample. Right: damage evolution of the fibrous network during the tensile test using the 3D FE model of Natali et al.'s ${ }^{16}$ experimental sample. 


\section{2 | PDL material model application to a canine microCT FE model}

After characterization of the PDL tissue behaviour, the porous fibrous hyperelastic damage model was implemented into the periodontium of a human canine FE model. In this section, the results of the FE model under three loading conditions are explained. Figure 7 shows the minimum principal stresses in the bone, the void ratio, damage of the fibrous network and the minimum principal stresses in the ligament after the first cycle under high strain rate. The damage evolution with the cycles is negligible in a damage-time curve at high strain rates (Figure 8A). Therefore, a local magnification of each damage-time curve is shown in detail in Figure $8 \mathrm{~A}$. Furthermore, the difference between the initial and final position of the tooth (named residual displacement) is shown in Figure 8B. In this figure, the differences regarding whether the damage phenomenon is considered at high or low strain rates is shown. The complete information of the stress distribution for the different tissues involved is provided in the available supplementary material.

\subsection{1 | Intrusive loading}

It is well known that the physiological role of the PDL is to absorb intrusive loads. When the intrusive load is applied, the tooth is displaced downward (see Appendix 1, Figure A1-iii), and the apical region of the PDL is compressed toward the bone (Figure 7A). Compression of the ligament causes the fluid to flow to the bone, decreasing the void ratio in this region (Figure 7B). The dissipation of fluid partially relaxes the ligament and contributes to bone loading. However, a large compression of the PDL may cause a reduction in the fluid content and an increase in the load supported by the solid phase. Due to the high loss of fluid in the apical region, the maximum value of the minimum principal stress is located in this region (Figure 7D). On the other hand, the void ratio of the alveolar crest region increases because the PDL is stretched in this region (Figure 7B).

Regarding the fibrous network role, the PDL is subjected to shear forces. The regions where the fibre bundles run horizontally and obliquely become more stretched, causing higher maximum principal stress in these areas (see Appendix 1, Figure A2-i). This effect increases the strain level in this region, causing damage to the fibrous network on the mesial side of the PDL (Figure 7C). Curiously, no fibrous damage appears at the top region of the ligament. Despite the excessive magnitude of the load, the maximum damage value is 0.29 and minimally increases by load repetition (see the continuous line in Figure 8A). In the case of slow load application, the fluid does not absorb the load, and the solid matrix supports most of it. In this case, the residual displacement increases (shown percentage differences in Figure $8 \mathrm{~B}$ legend) due to greater damage to the fibrous network. However, the PDL matrix is the least damaged of the three cases (Figure $8 \mathrm{~B}$ ) because fibres are primarily oriented to resist intrusive loads.

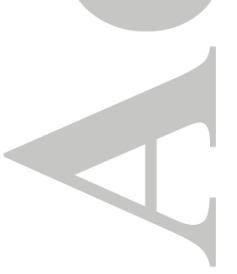




\subsection{2 | Labial-lingual loading}

The results of the FEA suggest that the tooth rotates when a lateral labial-lingual load is applied (see Appendix 1, Figure A1-iii). For this particular model, the rotation centre (RC) is at a depth of approximately $7 \mathrm{~mm}$ from the upper border of the ligament. This rotation movement causes compression efforts in the upper region of the opposite side of the bone (Figure 7A). Thus, the top region of the lingual side and the bottom region of the labial side of the ligament are compressed (Figure 7D), while the opposite regions are stretched (see Appendix 1, Figure A2-i). The compression reduces the void ratio (Figure 7B) in these sections, which increases the value of the minimum principal stresses in the solid matrix (Figure 7D) and the pressure of the interstitial fluid (see Appendix 1, Figure A2-iii).

On the other hand, the highest values of the maximum principal stresses are located at the top region of the labial side and at the bottom region of the lingual side (see Appendix 1, Figure A2-ii). Although similar displacement occurs in both regions, the apical region experiences higher stresses due to the small thickness of the PDL in this region. Thus, the damage of the fibres network starts at this region (Figure 7C). In this case, the maximum damage values $(83 \%)$ are similar to the total damage of the tissue. However, the damage evolution increases slowly within the cycles (Figure 8A) because the porous term continues to support much of the stress. For low strain rates, the fluid phase only minimally contributes, and the irreversible displacement due to fibrous damage increases (Figure 8B). This residual displacement percentage is related to the maximum damage value.

\subsubsection{Mesial-distal loading}

Loading along the mesial-distal direction is probably the most unusual loading scenario. The thinnest region of the PDL is located at the mesial-distal sides of the top region of the ligament since this region is minimally loaded under physiological conditions. As mentioned, the main role of the ligament is to resist the intrusive loads produced during chewing. When the tooth is subjected to loads along the mesial-distal direction, the tooth rotates (see Appendix 1, Figure A1-iii). This movement causes compression of the alveolar crest and apical regions of the distal and mesial sides, respectively (Figure 7B). Therefore, it reduces the fluid volume (Figure 7B) and increases the pressure of the fluid in the areas mainly compressed (see Appendix 1, Figure A2-iii). In addition, the fluid flow contributes to loading the underlying trabecular bone (Figure 7A). It is remarkable that the top region of the PDL at the distal side evacuates all the interstitial fluid of the PDL (Figure 7B). Thus, the maximum value of minimum principal stresses is located at the top region of the bone. On the other hand, the maximum value of maximum principal stresses is located at the apical region of the PDL distal side (see Appendix 1, Figure A2-A). The apical fibres must support high stress values in this region, causing damage to its fibrous structure. The damage value during mesial-distal loading is the highest of the three performed tests (Figure 8A) and may be caused by the unusual direction of the load. 
(A)
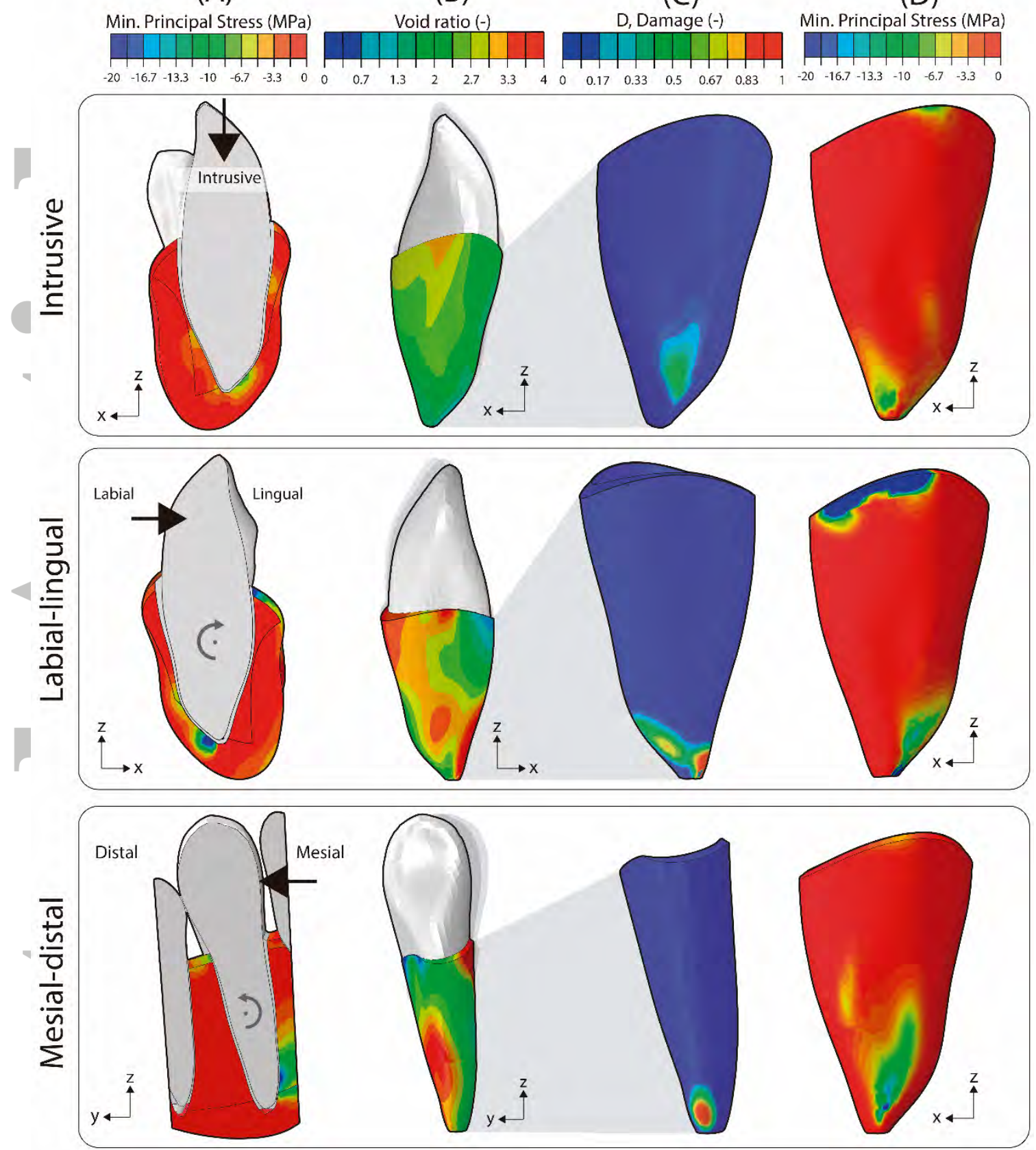

FIGURE 7 Top to bottom: FE results corresponding to the first cycle of applying a $360 \mathrm{~N}$ load in intrusive, labial-lingual and mesial-distal directions. (A) Minimum principal stresses in the cortical and trabecular bone portions, (B) Void ratio in the canine PDL, (C) Damage of the fibrous network of the PDL due to excessive stretch, (D) Minimum principal stresses in the canine PDL.

This article is protected by copyright. All rights reserved. 

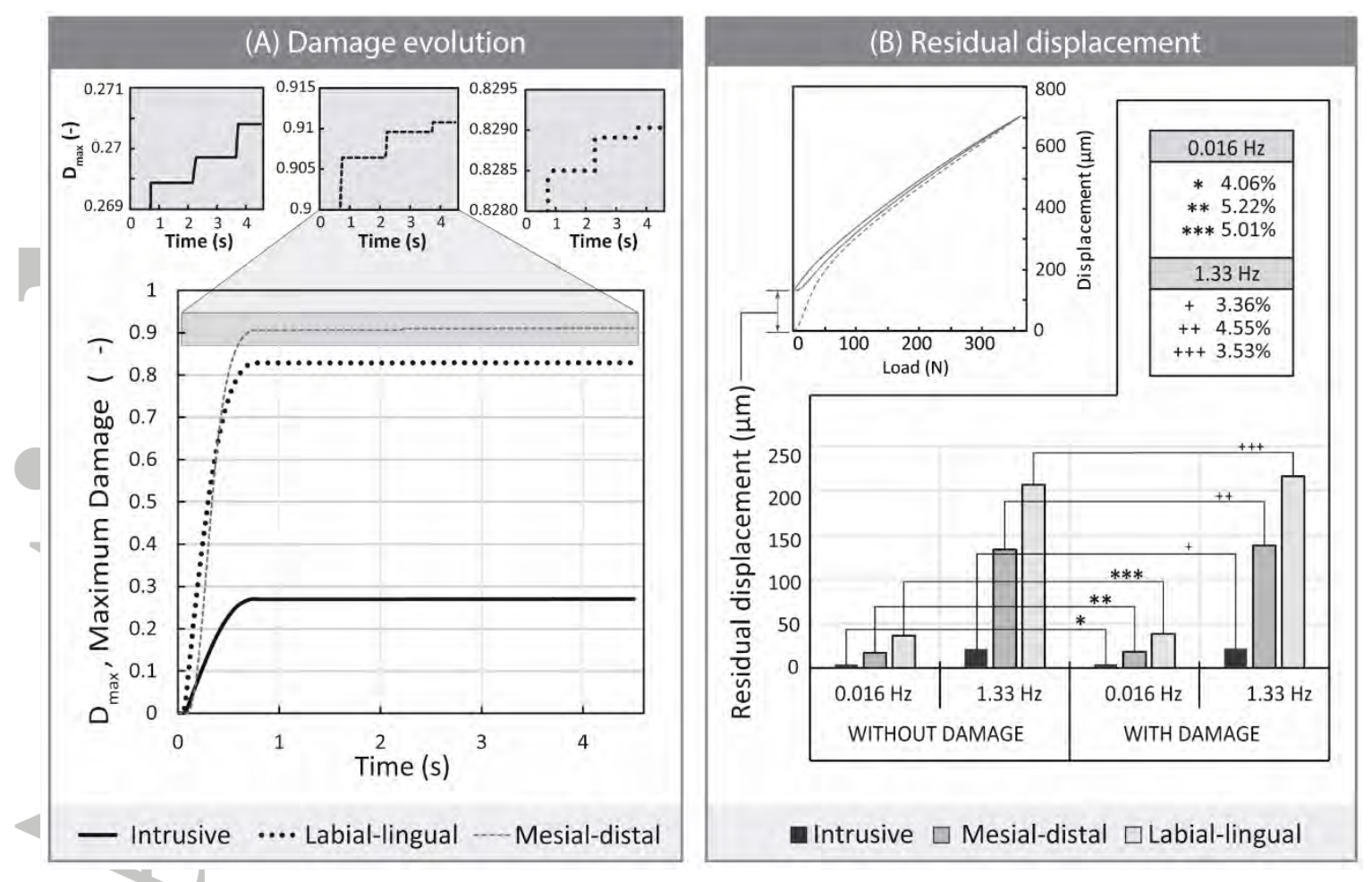

FIGURE 8 (A) Top: details of the damage evolution due to repetitive traumatic loading; bottom: damage evolution of the maximum damaged element for each loading scenario at 1.33 Hz. (B) Top: Load-displacement curve obtained by applying the load in the mesial-distal direction to show the concept of residual displacement; bottom: comparison of residual displacement at slow $(0.0166 \mathrm{~Hz})$ and fast $(1.33 \mathrm{~Hz})$ velocities, considering the non-damage or damage phenomenon (* Difference percentages at slow velocity, + Difference percentages at fast velocity).

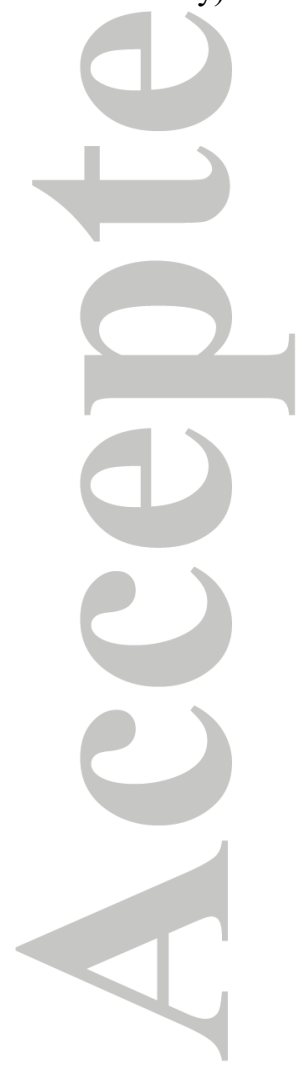

This article is protected by copyright. All rights reserved. 


\section{4 | DISCUSSION}

\subsection{Characterization and validation of the material model}

As mentioned in the introduction section, several studies have explained PDL behaviour using elastic isotropic or hyperelastic material formulations. ${ }^{23,25,26}$ Moreover, some computational studies have attempted to explain the non-linear anisotropic behaviour by introducing collagen fibres as 1D FE elements. ${ }^{61}$ On the other hand, some researchers have explained the effect of the PDL interstitial fluid by means of viscoelastic material formulations. ${ }^{12,27,28} \mathrm{We}$ recently proposed a new model that combines the porous and fibrous properties of the PDL. ${ }^{24}$ However, the role of the PDL in some of the most common periodontal diseases is unknown. This work is the first to study the porous fibrous behaviour of the PDL under traumatic loads. We suggest an innovative material model, namely, a porous fibrous material model, that considers the PDL softening behaviour due to fibre alignment and damage due to fibre rupture.

The first part of section 2.1 summarizes the material model without damage mechanism proposed in our previous work. As shown in Figure 4, good correlation exists between the numerical and experimental data under tensile and compressive efforts (Figures 4A and 4B, respectively). The tensile response of the PDL was characterized using experimental data of porcine samples from the literature, ${ }^{28}$ while the compressive behaviour was defined by data of bovine samples. ${ }^{14}$ Although the porcine PDL seems more similar to the human PDL, it has been suggested that no significant differences exist among the compressive responses of different species. ${ }^{28}$ This similarity was verified by subjecting the $3 \mathrm{D}$ FE model (which reproduces the experimental sample geometry in Natali's study) to Nishihira et al. ${ }^{52}$ loading scenarios where samples of an adult cat PDL were tested. The computational compressive response (characterized by bovine experimental data) was not significantly different from the Nishihira experimental results (Figure 4C). Remarkably, higher differences appeared at low strain rates $(0.0052$ and $0.55 \% / \mathrm{sec})$, when the porous component has minimal contributions. Thus, it can be assumed that the porous component is similar among different species and plays a key role under compressive efforts. In summary, the findings of the material model without damage demonstrated the biphasic behaviour of the tissue, as suggested by other authors. ${ }^{5,6,62}$ This study suggests that the stiffness highly depends on the fibrous structure (Figure 4A) under tensile forces and on the strain rate under compressive forces (Figure 4B).

Regarding the softening mechanisms, this study numerically demonstrated the softening effect due to fibre alignment and damage due to fibre rupture (Figure 2). The softening behaviour observed in Natali et al. ${ }^{29}$ was extrapolated to the experimental data of their later study. ${ }^{28}$ The Mullins effect was characterized using this theoretical assumption. Thus, the numerical results suggested a small softening effect of the fibrous network due to the alignment of fibres with repetitive loading (Figure 5A). The dissipated energy increased when the fibres ran along the loading direction (Figure $5 \mathrm{C}$ ) because the fibrous term has a greater influence on the PDL behaviour. Additionally, the softening effect under compressive forces can be seen in Figure 5C. Because " $n$ " is a loading-unloading cycle, the unloading curve of the " $n$ " cycle did not fit with the loading curve of the " $n+1$ " cycle (see details in Figures 5C and 5D) due to the viscoelastic contribution. Interestingly, the material model without a damage criterion did not provide as good a fit of the experimental data for strains 
less than 0.4 (Figure 4A). However, the softening phenomenon improved the fitting of the experimental data (Figure 5A). This improvement could mean that the specimens tested by Natali et al. ${ }^{28}$ were also previously preconditioned.

Regarding fibrous network damage, the experimental test performed by Natali et al. ${ }^{28}$ was computationally mimicked (Figure 6B). For strains higher than 0.6 , the numerical response of the material model without damage was stiffer than the experimental data. According to our material description, this deviation could be caused by fibrous network damage.

\subsection{PDL material model application to the microCT FE model}

The causes of chronic periodontitis have been mainly explained from an infectious point of view. The infection can begin with an inflammatory response due to a repetitive traumatic load. Thus, interest in studying the role of the mechanical response of the PDL in periodontitis diseases has increased in recent years. ${ }^{2,3,62}$ The PDL plays two roles under traumatic loading conditions. First, it works as a mechanical stimulus receptor and informs the chewing system when overload occurs; then, the chewing muscles immediately reduce the loads. On the other hand, the PDL releases inflammatory mediators that cause bone remodelling. Therefore, several authors ${ }^{4,40}$ suggest that the PDL plays a key role in tooth movement, and some studies have sought to explain bone resorption according to its mechanical response. ${ }^{63,64}$

Figure 7A shows the regions of the cortical and trabecular bones that are mainly compressed. For lateral loads (labial-lingual and mesial-distal directions), the highest minimum principal stresses were observed at the alveolar crestal and apical regions of the PDL. The compressive stresses in the alveolar crest zone are caused by rotation of the tooth and coincide with the middle region of the tooth (see Appendix 1, Figures A1-iii). On the other hand, the value of the minimum principal stresses is maximum in the apical zone due to the interaction of two effects. First, compression of the PDL decreases the fluid volume in that area, causing an increase in the load supported by the fibrous network. This overloading state damages the matrix and causes a weakening of the PDL. This fact can be seen in Figure 8B, which shows greater damage for low strain rates due to the small influence of the fluid. Second, the maximum value of the minimum principal stress in the apical region is caused by the increase in fluid pressure with cycles and by the softening phenomena.

Both softening phenomena reduce the stiffness of the solid matrix. This fact increases the interstitial liquid pressure at the regions that are mainly compressed (see Appendix 1, Figure A2-iii). Some of the most common bone remodelling hypotheses of tooth movement are that tensile strain of the ligament causes the formation of bone tissue, while compressive strain causes the resorption of bone tissue. We refer to the "pressure-tension hypothesis", 65,66 "alveolar bending hypothesis" ${ }^{67}$ or "stretched fibre hypothesis", 68 among others. This study suggests that mandibular bone remodelling cannot be explained in simple terms of tension or compression regions. We considered that a complete study of the porous fibrous structure of the PDL should be performed to explain the bone remodelling mechanism. Based on our numerical results, we predict that the bone remodelling mechanics may be a combination of 
the "stretched fibre hypothesis", damage to the fibrous network and overpressure of the interstitial fluid.

From a cellular point of view, recent studies have considered that bone resorption could be caused by the combination of three mechanisms. ${ }^{69,70}$ First, the fibres compressed at the alveolar crest or apical regions (Figure 7A) could release some cytokines and prostaglandins to form leucocytes, macrophages, and monocytes. ${ }^{70}$ Then, the chemical changes at these compressive regions may recruit and differentiate cells into osteoclasts. These osteoclasts would reabsorb the adjacent bone and destroy the necrotic hyalinized tissue. ${ }^{71}$ On the other hand, overpressure of the interstitial fluid (Figure 7B) can produce inflammatory cytokines. ${ }^{72}$ Cytokines also stimulate bone resorption. ${ }^{73}$ Finally, it is well known that the adjacent bone contains fenestrations, ${ }^{74}$ which can detect whether the PDL fluid is compressed. ${ }^{75,76}$ Stimulation by fenestrations reduces the bone density. ${ }^{75-77}$

By contrast, the formation of bone tissue can be explained by fibre stretching. ${ }^{78-80}$ The maximum value of the maximal principal stresses can be found in the apical region of the PDL (see Appendix 1, Figure A2-i). The over-elongation of fibres in this region causes fibrous network damage (Figure 7C), local vascularization ${ }^{70}$ and mechanobiological signals (arachidonic acid metabolites, neuropeptides or cytokines). ${ }^{81-83}$ These signals can translate to bone formation around these sides of the ligament. However, excessive stretching of fibres can cause PDL deterioration (Figure $7 \mathrm{C}$ ) ${ }^{16}$ and trigger inflammatory processes. ${ }^{84}$ In this case, cytokines may initiate the bone resorption process.

Furthermore, the fibre contribution under intrusive loads causes a well-balanced stress distribution along the PDL (Figure 7A). ${ }^{4}$ This uniform stress state can prevent bone resorption, especially in the middle region. Biologically, fenestrations of the bone in this region may not receive enough mechanical signals to initiate bone formation. ${ }^{74}$ In our simulations, only a small area of the ligament experiences damage to the fibrous network (Figure 7C), and the maximum damage value in this case is smaller than in the other two cases. According to the bone remodelling principles followed in this study ("stretched fibre theory", fluid pressure and PDL damage mechanism), bone resorption can begin at the apical region since the previously mentioned rules are satisfied. However, the uniform mechanical stress state maintains stability of the bone density. 


\section{3 | Study limitations}

There are several limitations of the current study, which future research should address. The most important limitation lies in the fact that experimental data of human PDL samples were not used. Nevertheless, experimental data of porcine and bovine PDL samples have been extensively used because their bite systems have mechanical similarities with the human bite system. Moreover, the same animal species was not used for the compressive and tensile behaviour characterization. Future experimental studies should test porcine samples under compressive and tensile efforts, considering the fibrous network and the permeability of the tissue.

Second, the Mullins effect and the tensile material response were characterized using experimental data from different studies (Natali et al. ${ }^{16}$ and Natali et al., ${ }^{28}$ respectively). Although both studies were performed by the same authors, the Mullins effect was characterized by a theoretical curve. On the other hand, this material model only uses one function to describe both the matrix and fibre alignment softening phenomena. Experimental tests should be performed to separately determine the effects of repetitive loading on the matrix and the fibrous network.

Another major source of uncertainty is the experimental data used to characterize the damage of the fibrous network. It was assumed that the damage process started at 0.6 strain, but this assumption is not based on histological experimental studies. Fibrous damage should be studied by a combination of microscopic and macroscopic analysis. Advances in these two areas would improve our knowledge of the complex degradation mechanism of the PDL.

Although this study verifies the important effect of fibrous damage, damage due to overpressure of the interstitial fluid was not considered. To take into account this effect, other authors ${ }^{85}$ have simplified the fluid pressure by the volume averaged hydrostatic pressure, $\bar{\sigma}_{h}$ defined as $\bar{\sigma}_{H}=\frac{\sum_{e} \sigma_{H}^{e} V^{e}}{\sum_{e} V^{e}}$, where $\sigma_{H}^{e}$ and $V^{e}$ are the hydrostatic stress and volume of each element. They consider that bone regeneration occurs for higher $\bar{\sigma}_{h}$ values than the capillary blood pressure $\bar{\sigma}_{h}=4.7 \mathrm{kPa}(35 \mathrm{mmHg})$. However, this is a severe assumption because it does not consider the real viscoelastic effect of the fluid phase. We have to remark that the fluid effect must be considered in future studies to acquire complete knowledge of the PDL. Based on mechanobiological studies of PDL degeneration, the fluid phase likely plays the most significant role in PDL diseases.

Finally, using FE models to study patient-specific periodontal diseases from a practical point of view will require extensive further development. This study was developed using a computational model for a specific patient tooth, and the numerical results cannot be directly extrapolated to other cases. However, the FE model presented in this manuscript contributes to improving our knowledge of the role of the PDL in supporting loads. 


\section{5 | CONCLUSIONS}

The present results demonstrate the applicability of a porous fibrous damage hyperelastic material model to describe the mechanical response of the PDL and its damage under traumatic conditions. Within the limitations of this study, we summarize our findings by the following conclusions:

1. Both porous and fibrous effects must be considered in numerical studies of periodontium diseases. In particular, these contributions must be included in studies that analyse the stress state of the ligament and its effect on bone remodelling.

2. The preconditioning process modifies the ligament response, reducing its stiffness in mechanical experimental tests. Furthermore, this softening effect increases the residual displacement of the tooth under intrusive and lateral loads, which are common loads during chewing or parafunctional clenching.

3. The damage produced by fibre stretching occurs at high strains (upper than $60 \%$ ). The PDL fibrous network is not damaged under physiological loading scenarios. However, it may suffer small deterioration under traumatic loads, as studied in this manuscript.

4. The fluid pressure in the alveolar crest and apical regions may cause a deterioration process due to the interstitial fluid overpressure under lateral and intrusive traumatic loads, respectively. The effect of overpressure may be the main cause of deterioration of a healthy PDL under non-physiological loads.

5. The interstitial fluid effect, the collagen bundles and the softening phenomena should be simultaneously considered to explain the bone remodelling mechanism.

This study demonstrates the key roles of interstitial fluid and collagen fibres in the tissue mechanical response, the softening phenomenon due to fibre alignment or fibrous network damage, and the effect of considering a non-uniform thickness of the PDL by microCT scan techniques. The results presented here will serve as a basis for future studies and will help elucidate the role of the PDL in some of the most common periodontal diseases or bone remodelling processes. This FE model marks the beginning of potential research on PDL mechanical behaviour. Future studies should consider PDL damage due to overpressure of the interstitial fluid, the role of the PDL in real chewing cases, its deterioration under parafunctional loads, or the rates of bone remodelling in the mandible.

\section{ACKNOWLEDGEMENTS}

This work was supported by the Spanish Ministry of Economy and Competitiveness through project DPI 2016-79302-R.

\section{CONFLICT OF INTEREST}

The authors have no conflicts of interest related to the present work.

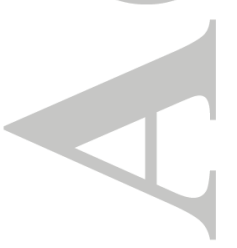




\section{REFERENCES}

1. Poiate, I. A. V. P., de Vasconcellos, A. B., de Santana, R. B. \& Poiate, E. ThreeDimensional Stress Distribution in the Human Periodontal Ligament in Masticatory, Parafunctional, and Trauma Loads: Finite Element Analysis. Journal of Periodontology. 2009. 80, 1859-1867. doi:10.1902/jop.2009.090220.

2. Pihlstrom, B. L., Michalowicz, B. S. \& Johnson, N. W. Periodontal diseases. Lancet (London, England). 2005. 366, 1809-20. doi:10.1016/S0140-6736(05)67728-8.

3. Drolshagen, M., Keilig, L., Hasan, I., et al. Development of a novel intraoral measurement device to determine the biomechanical characteristics of the human periodontal ligament. Journal of Biomechanics. 2011. 44, 2136-2143. doi: 10.1016/j.jbiomech.2011.05.025.

4. Tuna, M., Sunbuloglu, E. \& Bozdag, E. Finite element simulation of the behavior of the periodontal ligament: A validated nonlinear contact model. Journal of Biomechanics. 2014. 47, 2883-2890. doi:10.1016/j.jbiomech.2014.07.023.

5. Favino, M., Gross, C., Drolshagen, M., et al. Validation of a heterogeneous elasticbiphasic model for the numerical simulation of the PDL. Computer Methods in Biomechanics and Biomedical Engineering. 2013 16, 544-553. doi:10.1080/10255842.2011.628660.

6. Bergomi, M., Cugnoni, J., Botsis, J., et al. The role of the fluid phase in the viscous response of bovine periodontal ligament. Journal of Biomechanics. 2010. 43, 1146-1152. doi:10.1016/j.jbiomech.2009.12.020.

7. Daly, C. H., Nicholls, J. I., Kydd, W. L. \& Nansen, P. D. The response of the human periodontal ligament to torsional loading-I. Experimental methods. Journal of Biomechanics. 1974. 7, 517-522. doi:10.1016/0021-9290(74)90086-4.

8. Berkovitz, B. K. B., Weaver, M. E., Shore, R. C. \& Moxham, B. J. Fibril Diameters in the Extracellular Matrix of the Periodontal Connective Tissues of the Rat. Connective Tissue Research. 1981. 8, 127-132. doi: 10.3109/03008208109152132.

9. Tsuzuki, T., Kajiya, H., T-goto, K. \& Tsutsumi, T. Hyperocclusion stimulates the expression of collagen type XII in periodontal ligament. Archives of Oral Biology. 2016. 66, 86-91. doi:10.1016/j.archoralbio.2016.02.009.

10. Qian, L., Todo, M., Morita, Y., Matsushita, Y. \& Koyano, K. Deformation analysis of the periodontium considering the viscoelasticity of the periodontal ligament. Dental materials : official publication of the Academy of Dental Materials. 2009. 25, 1285-92. doi:10.1016/j.dental.2009.03.014.

11. Toms, S. R., Dakin, G. J., Lemons, J. E. \& Eberhardt, A. W. Quasi-linear viscoelastic behavior of the human periodontal ligament. Journal of Biomechanics. 2002. 35, 14111415. doi:10.1016/S0021-9290(02)00166-5.

12. Chen-Ying, W., Ming-Zen, Su., Hao-Hueng, C., et al. Tension-compression viscoelastic behaviors of the periodontal ligament. Journal of the Formosan Medical Association. 2012. 111, 471-481. doi:10.1016/j.jfma.2011.06.009.

13. Nyashin, M. Y., Osipov, A. P., Bolotova, M. P., Nyashin, Y. I. \& Simanovskaya, E. Y. Periodontal ligament may be viewed as a porous material filled by free fluid: experimental proof. Russian Journal Biomechanics. 1999. 3, 89-95 89-95. 
14. Bergomi, M., Cugnoni, J., Galli, M., Botsis, J., Belser UC. \& Wiskott HW. Hydromechanical coupling in the periodontal ligament: A porohyperelastic finite element model. Journal of Biomechanics. 2011. 44, 34-38. doi:10.1016/j.jbiomech.2010.08.019.

15. Natali, A. N., Pavan, P. G., Venturato, C. \& Komatsu, K. Constitutive modeling of the non-linear visco-elasticity of the periodontal ligament. Computer Methods and Programs in Biomedicine. 2011. 104, 193-198. doi:10.1016/j.cmpb.2011.03.014.

16. Natali, A. N., Pavan, P. G., Carniel, E. L. \& Dorow, C. A Transversally Isotropic Elastodamage Constitutive Model for the Periodontal Ligament. Computer Methods in Biomechanics and Biomedical Engineering. 2003. 6, 329-336. doi:10.1080/10255840310001639840.

17. Fill, T. S., Carey, J. P., Toogood, R. W. \& Major, P. W. Experimentally Determined Mechanical Properties of, and Models for, the Periodontal Ligament: Critical Review of Current Literature. Journal of Dental Biomechanics. 2011. 2, 312980-312980. doi: $10.4061 / 2011 / 312980$.

18. Detamore, M. S. \& Athanasiou, K. A. Tensile properties of the porcine temporomandibular joint disc. Journal of biomechanical engineering. 2003. 125, 558565. doi: 10.1115/1.1589778.

19. Genna, F., Annovazzi, L., Bonesi, C., Fogazzi, P. \& Paganelli, C. On the experimental determination of some mechanical properties of porcine periodontal ligament. 2008. Meccanica 43, 55-73. doi: 10.1007/s11012-007-9094-2.

20. Kalpakci, K. N., Willard, V. P., Wong, M. E. \& Athanasiou, K. A. An Interspecies Comparison of the Temporomandibular Joint Disc. Journal of Dental Research. 2011. 90, 193-198. doi:10.1177/0022034510381501.

21. Chang, C., Lei, Y., Ho, Y., Sung, Y. \& Lin, T. Predicting the holistic force-displacement relation of the periodontal ligament: in-vitro experiments and finite element analysis. Biomedical engineering online. 2014. 13.1, 107. doi:10.1186/1475-925X-13-107.

22. Benazzi, S., Nguyen, H. N., Kullmer, O. \& Kupczik, K. Dynamic Modelling of Tooth Deformation Using Occlusal Kinematics and Finite Element Analysis. Plos one. 2016. 117. doi:10.1371/journal.pone.0152663.

23. Nikolaus, A., Fleck, C., Lindtner, T., Currey, J. \& Zaslansky, P. Importance of the variable periodontal ligament geometry for whole tooth mechanical function: A validated numerical study. Journal of the Mechanical Behavior of Biomedical Materials. 2016. 67, 61-73. doi:10.1016/j.jmbbm.2016.11.020.

24. Ortún-Terrazas, J., Cegoñino, J., Santana-Penín, U., Santana-Mora, U. \& Pérez del Palomar, A. Approach towards the porous fibrous structure of the periodontal ligament using micro-computerized tomography and finite element analysis. Journal of the Mechanical Behavior of Biomedical Materials. 2018. 79, 135-149. doi:10.1016/j.jmbbm.2017.12.022.

25. Zhang, Z. et al. Mechanical benefits of conservative restoration for dental fissure caries. Journal of the Mechanical Behavior of Biomedical Materials. 2016. 53, 11-20. doi:10.1016/j.jmbbm.2015.08.010.

26. Oskui, I. Z., Hashemi, A. \& Jafarzadeh, H. Biomechanical behavior of bovine periodontal ligament: Experimental tests and constitutive model. Journal of the Mechanical Behavior of Biomedical Materials. 2016. 62, 599-606. doi:10.1016/j.jmbbm.2016.05.036. 
27. Huang, H., Tang, W., Tan, Q. \& Yan, B. Development and parameter identification of a visco-hyperelastic model for the periodontal ligament. Journal of the Mechanical Behavior of Biomedical Materials. 2017. 68, 210-215. doi:10.1016/j.jmbbm.2017.01.035.

28. Natali, A. N. et al. A visco-hyperelastic-damage constitutive model for the analysis of the biomechanical response of the periodontal ligament. Journal of biomechanical engineering. 2008. 130, 31004. doi:10.1115/1.2900415.

29. Natali, A. N., Pavan, P. G., Carniel, E. L. \& Dorow, C. Viscoelastic Response of the Periodontal Ligament: An Experimental - Numerical Analysis. Connect. Tissue Res. 2004. 222-230. doi:10.1080/03008200490885742

30. Fill, T. S., Toogood, R. W., Major, P. W. \& Carey, J. P. Analytically determined mechanical properties of, and models for the periodontal ligament: Critical review of literature. J. Biomech. 2012. 45, 9-16. 10.1016/j.jbiomech.2011.09.020

31. Hirashima, S., Ohta, K., Kanazawa, T., Okayama, S., Togo, A., Miyazono, Y., ... \& Nakamura, K. I. Three-dimensional ultrastructural analysis and histomorphometry of collagen bundles in the periodontal ligament using focused ion beam/scanning electron microscope tomography. J. Periodontal Res. 2018. 1-9. doi:10.1111/jre.12592

32. Maher, E., Creane, A., Lally, C. \& Kelly, D. J. An anisotropic inelastic constitutive model to describe stress softening and permanent deformation in arterial tissue. J. Mech. Behav. Biomed. Mater. 2012. 12, 9-19. doi: https://doi.org/10.1016/j.jmbbm.2012.03.001

33. Alastrué, V., Peña, E., Martínez, M. A. \& Doblaré, M. Experimental study and constitutive modelling of the passive mechanical properties of the ovine infrarenal vena cava tissue. J. Biomech. 2008. 41, 3038-3045. doi: 10.1016/j.jbiomech.2008.07.008

34. Peña, E. et al. Experimental study and constitutive modeling of the viscoelastic mechanical properties of the human prolapsed vaginal tissue. Biomech. Model. Mechanobiol. 2010. 9, 35-44. doi: 10.1007/s10237-009-0157-2

35. Natali, A. N., Carniel, E. L. \& Gregersen, H. Biomechanical behaviour of oesophageal tissues: material and structural configuration, experimental data and constitutive analysis.

Med. Eng. Phys. 2009. 31, 1056-1062. doi: 10.1016/j.medengphy.2009.07.003

36. Storakers, B. On material representation and constitutive branching in finite compressible elasticity. 1986. J. Mech. Phys. Solids 34, 125-145. doi: 10.1016/0022-5096(86)90033-5

37. $\mathrm{Wu}, \mathrm{T}$. H. Soil mechanics. 1967.

38. Argoubi, M. \& Shirazi-Adl, A. Poroelastic creep response analysis of a lumbar motion segment in compression. Journal of Biomechanics. 1996. 29, 1331-1339. doi:10.1016/0021-9290(96)00035-8.

39. Natali, A. N., Pavan, P. G., Schrefler, B. A. \& Secchi, S. A multi-phase media formulation for biomechanical analysis of periodontal ligament. Meccanica. 2002. 37, 407-418.doi: 10.1023/A:1020895906292

40. Dorow, C., Krstin, N. \& Sander, F.-G. Experiments to Determine the Material Properties of the Periodontal Ligament. Journal of Orofacial Orthopedics / Fortschritte der Kieferorthopädie. 2002. 63, 94-104. doi: 10.1007/s00056-002-0107-4.

41. Pini, M., Zysset, P., Botsis, J. \& Contro, R. Tensile and compressive behaviour of the bovine periodontal ligament. Journal of Biomechanics. 2004. 37, 111-119. doi:10.1016/S0021-9290(03)00234-3. 
42. Bergomi, M., Anselm Wiskott, H. W., Botsis, J., Shibata, T. \& Belser, U. C. Mechanical response of periodontal ligament: Effects of specimen geometry, preconditioning cycles and time lapse. Journal of Biomechanics. 2009. 42, 2410- 2414. doi:10.1016/j.jbiomech.2009.06.031.

43. Mullins, L. Effect of Stretching on the Properties of Rubber. Rubber Chemistry and Technology. 1948. 21, 281-300. doi: 10.5254/1.3546914.

44. Ehret, A. E. \& Itskov, M. Modeling of anisotropic softening phenomena: Application to soft biological tissues. International Journal of Plasticity. 2009. 25, 901-919. doi:10.1016/j.ijplas.2008.06.001.

45. Ogden, R. W. \& Roxburgh, D. G. A pseudo-elastic model for the Mullins effect in filled rubber. Proceedings of the Royal Society A: Mathematical, Physical and Engineering Sciences. 1999. 455, 2861-2877. doi:10.1098/rspa.1999.0431.

46. Horgan, C. O., Ogden, R. W. \& Saccomandi, G. A theory of stress softening of elastomers based on finite chain extensibility. Proceedings of the Royal Society A: Mathematical, Physical and Engineering Sciences. 2004. 460, 1737-1754. doi:10.1098/rspa.2003.1248.

47. Provenzano, P. P., Heisey, D., Hayashi, K., Lakes, R. \& Vanderby, R. Subfailure damage in ligament: a structural and cellular evaluation. Journal of Applied Physiology. 2002. 92, 362-371. doi:10.1152/jappl.2002.92.1.362.

48. Huang, H., Tang, W., Yan, B. \& Wu, B. Mechanical responses of periodontal ligament under a realistic orthodontic loading. Procedia Engineering. 2012. 31, 828-833. doi:10.1016/j.proeng.2012.01.1108.

49. Boryor, A. et al. A downloadable meshed human canine tooth model with PDL and bone for finite element simulations. Dental Materials. 2009. 25, 57-62. doi:10.1016/j.dental.2009.05.002.

50. Peña, E., Calvo, B., Martínez, M. A. \& Doblaré, M. On finite-strain damage of viscoelastic-fibred materials. Application to soft biological tissues. Int. J. Numer. Methods Eng. 2008. 74, 1198-1218.doi: 10.1002/nme.2212

51. Rodríguez, J. F., Cacho, F., Bea, J. A. \& Doblaré, M. A stochastic-structurally based three dimensional finite-strain damage model for fibrous soft tissue. Journal of the Mechanics and Physics of Solids. 2006. 54, 864-886. doi:10.1016/j.jmps.2005.10.005.

52. Nishihira, M., Yamamoto, K., Sato, Y., Ishikawa, H. \& Natali, A. Mechanics of periodontal ligament. In Dental Biomechanics. 2003. $1^{\text {st }}$ ed. 20-32.

53. Belli, S., Eraslan, O. \& Eskitaşcioğlu, G. Effect of Different Treatment Options on Biomechanics of Immature Teeth: A Finite Element Stress Analysis Study. Journal of Endodontics. 2017. doi:10.1016/j.joen.2017.08.037.

54. Razaghi, R., Biglari, H. \& Karimi, A. Dynamic finite element simulation of dental prostheses during chewing using muscle equivalent force and trajectory approaches. Journal of Medical Engineering \& Technology. 2017. 41, 314-324. doi:10.1080/03091902.2017.1299231.

55. Hattori, Y. et al. Bite forces and their resultants during forceful intercuspal clenching in humans. J. Biomech. 2009. 42, 1533-1538.doi: 10.1016/j.jbiomech.2009.03.040

56. Kerstein, R. B. \& Radke, J. Average chewing pattern improvements following Disclusion Time reduction. CRANIO®. 2017. 35, 135-151. doi:10.1080/08869634.2016.1190526. 
57. Zheng, J. \& Zhou, Z. R. Effect of age on the friction and wear behaviors of human teeth. Tribology International. 2006. 39, 266-273. doi:10.1016/j.triboint.2004.09.004.

58. Schünke, M. Prometheus-Lernatlas der Anatomie: Innere Organe; 118 Tabellen (Vol. 2). 2009 Georg Thieme Verlag.

59. Shu-Li, L., Sheng-Yang, L., Yen-Chuang, L., Yen-Hua, H., Jen-ChangY. \& Haw-Ming H. Evaluation of mechanical and histological properties of cryopreserved human premolars under short-term preservation: A preliminary study. Journal of Dental Sciences. 2014. 9, 244-248. doi:10.1016/j.jds.2013.04.010.

60. Lacroix, D. \& Prendergast, P. J. A mechano-regulation model for tissue differentiation during fracture healing: analysis of gap size and loading. Journal of Biomechanics. 2002. 35, 1163-1171. doi:10.1016/S0021-9290(02)00086-6.

61. Freitas, A. C., Rocha, EP., dos Santos, PH., Ko, CC., Martín, M. \& de Almeida, EO. Mechanics of the maxillary central incisor. Influence of the periodontal ligament represented by beam elements. Computer methods in biomechanics and biomedical engineering. 2010. 13, 515-21. doi:10.1080/10255840903273175.

62. Keilig, L., Drolshagen, M., Tran, K. L., Hasan, I., Reimann, S., Deschner, J., \& Bourauel, C. In vivo measurements and numerical analysis of the biomechanical characteristics of the human periodontal ligament. Annals of Anatomy - Anatomischer Anzeiger. 2016. 206, 80-88. doi:10.1016/j.aanat.2015.08.004.

63. Qian, H., Chen, J. \& Katona, T. R. The influence of PDL principal fibers in a 3dimensional analysis of orthodontic tooth movement. American Journal of Orthodontics and Dentofacial Orthopedics. 2001. 120, 272-279. doi:10.1067/mod.2001.116085.

64. Zargham, A., Geramy, A. \& Rouhi, G. Evaluation of long-term orthodontic tooth movement considering bone remodeling process and in the presence of alveolar bone loss using finite element method. Orthodontic Waves. 2016. 5, 1-12. doi:10.1016/j.odw.2016.09.001.

65. Schwarz, A. M. Tissue changes incidental to orthodontic tooth movement. International Journal of Orthodontia, Oral Surgery and Radiography 1932. 18, 331-352. doi:10.1016/S0099-6963(32)80074-8.

66. Reitan, K. The initial tissue reaction incident to orthodontic tooth movement as related to the influence of function; an experimental histologic study on animal and human material. Acta Odontol Scand. 1951. 6:1-240.

67. Baumrind, S. A reconsideration of the propriety of the 'pressure-tension' hypothesis. American Journal of Orthodontics. 1969. 55, 12-22. doi:doi.org/10.1016/S00029416(69)90170-5.

68. Melsen, B. Tissue reaction to orthodontic tooth movement--a new paradigm. European journal of orthodontics. 2001. 23, 671-81.

69. Kim, S.-J., Park, K.-H., Park, Y.-G., Lee, S.-W. \& Kang, Y.-G. Compressive stress induced the up-regulation of M-CSF, RANKL, TNF- $\alpha$ expression and the downregulation of OPG expression in PDL cells via the integrin-FAK pathway. Archives of oral biology. 2013. 58, 707-716. doi:10.1016/j.archoralbio.2012.11.003.

70. Dutra, E. H., Nanda, R. \& Yadav, S. Bone Response of Loaded Periodontal Ligament. Current Osteoporosis Reports. 2016. 14, 280-283. doi:10.1007/s11914-016-0328-X. 
71. Rosenstiel, S. F., Land, M. F., \& Fujimoto, J. Contemporary Fixed Prosthodontics-EBook. Elsevier Health Sciences. 2015. ISBN: 0323112889.

72. Goto, H. et al. Human bone marrow adipocytes support dexamethasone-induced osteoclast differentiation and function through RANKL expression. Biomedical research (Tokyo, Japan). 2011. 32, 37-44. doi:10.2220/biomedres.32.37.

73. Kapoor, P., Kharbanda, O. P., Monga, N., Miglani, R. \& Kapila, S. Effect of orthodontic forces on cytokine and receptor levels in gingival crevicular fluid: a systematic review. Progress in orthodontics. 2014. 15, 65. doi: 10.1186/s40510-014-0065-6.

74. Bright, R., Hynes, K., Gronthos, S. \& Bartold, P. M. Periodontal ligament-derived cells for periodontal regeneration in animal models: a systematic review. Journal of Periodontal Research. 2015. 50, 160-172. doi:10.1111/jre.12205.

75. Bien, S. M. Hydrodynamic damping of tooth movement. Journal of dental research. 1966. 45(3), 907-914. doi: 10.1177/00220345660450036701.

76. Bergomi, M., Wiskott, H. W. A., Botsis, J., Mellal, A. \& Belser, U. C. Load response of periodontal ligament: assessment of fluid flow, compressibility, and effect of pore pressure. Journal of biomechanical engineering. 2010. 132, 14504. doi:10.1115/1.4000154.

77. Guo, Z., Bo, D., He, P., Li, H., Wu, G., Li, Z., ... \& Li, Q.. Sequential controlled-released dual-drug loaded scaffold for guided bone regeneration in a rat fenestration defect model. Journal of Materials Chemistry B. 2017. 5(37), 7701-7710. doi:10.1039/C7TB00909G.

78. McCormack, S. W., Witzel, U., Watson, P. J., Fagan, M. J. \& Gröning, F. Inclusion of periodontal ligament fibres in mandibular finite element models leads to an increase in alveolar bone strains. PLoS ONE. 2017. 12, 1-23. doi:10.1371/journal.pone.0188707.

79. Bernick, $\mathrm{S}$. The organization of the periodontal membrane fibres of the developing molars of rats. Archives of oral biology. 1960. 2, 57-63. doi:10.1016/0003-9969(60)90038-8.

80. McCormack, S. W., Witzel, U., Watson, P. J., Fagan, M. J. \& Gröning, F. The biomechanical function of periodontal ligament fibres in orthodontic tooth movement. Plos one. 2014. vol. 9, no 7, p. e102387. doi:10.1371/journal.pone.0102387.

81. Yamamoto, T. T. Orthodontic tooth movement and expression of calcium regulating hormone. Clinical calcium. 2012. 22, 91-98.

82. Kashyap, S. Current concepts in the biology of orthodontic tooth movement: a brief overview. NJDSR. 2006. 1, 28-31. doi:10.1016/j.ajodo.2005.12.013.

83. Yamaguchi, M., Shimizu, N., Goseki, T., Shibata, Y., Takiguchi, H., Iwasawa, T., \& Abiko, Y. Effect of different magnitudes of tension force on prostaglandin E2 production by human periodontal ligament cells. Archives of oral biology. 1994. 39(10), 877-884. doi:10.1016/0003-9969(94)90019-1.

84. Hallmon, W. W. Occlusal trauma: effect and impact on the periodontium. Annals of periodontology / the American Academy of Periodontology. 1999. 4, 102-108. doi:10.1902/annals.1999.4.1.102.

85. Chen, J., Li, W., Swain, M. V., Ali Darendeliler, M. \& Li, Q. A periodontal ligament driven remodeling algorithm for orthodontic tooth movement. Journal of Biomechanics. 2014. 47, 1689-1695. doi:10.1016/j.jbiomech.2014.02.030.

86. Holzapfel, G. A. Nonlinear solid mechanics: a continuum approach for engineering science. Meccanica, 2002, 37(4), 489-490. 
87. Marsden, Jerrold E., and Thomas JR Hughes. Mathematical foundations of elasticity. Courier Corporation, 1994.

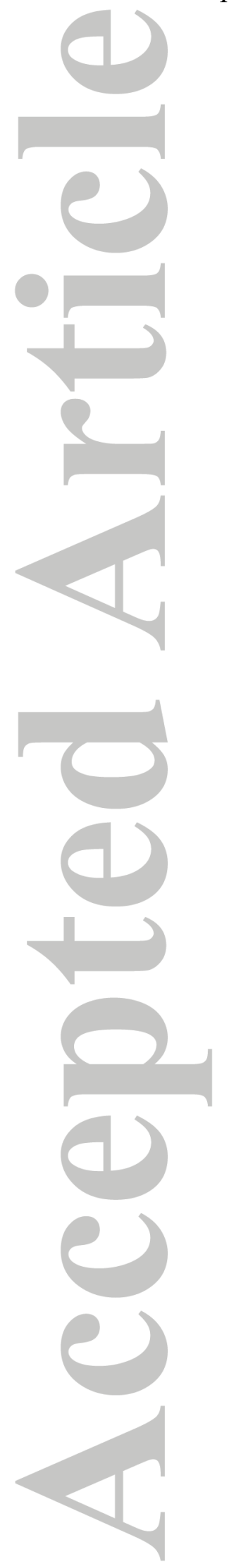




\section{APPENDIX 1- BIOMECHANICAL ANALYSIS OF THE TEETH, PDL AND MANDIBULAR BONE}

As it was introduced, the PDL works as a stress absorber to transmit loads from the tooth to the alveolar bone. Our numerical results verify that the PDL distributes intrusive loads better than lateral loads to the teeth and the mandibular bone. Under an intrusive load, the tooth moves vertically (Figure A1-iii), and homogeneous maximum and minimum principal stress distributions are generated along the tooth (Figure A1-i and A1-ii, respectively). On the other hand, the lateral loads cause tooth rotation (Figure A1-iii). This movement concentrates the higher stresses in the middle region of the tooth.

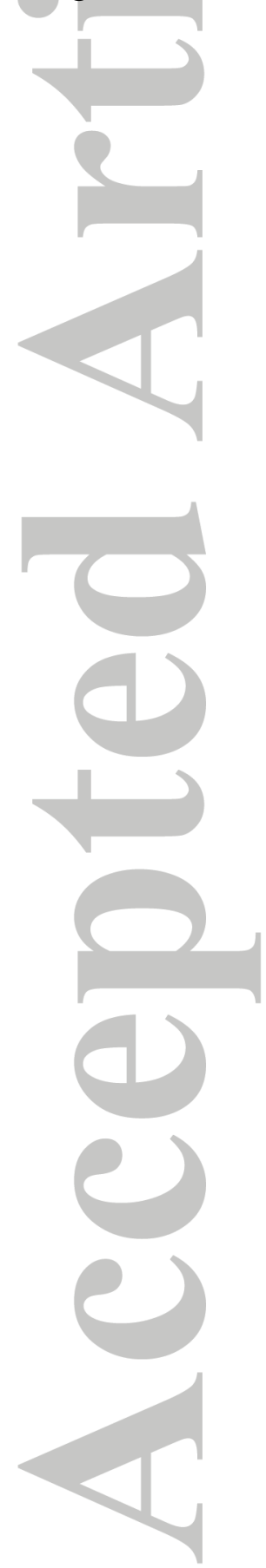


(i)

(ii)

Max. Principal Stress (MPa) Min. Principal Stress (MPa)

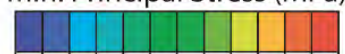

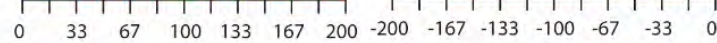
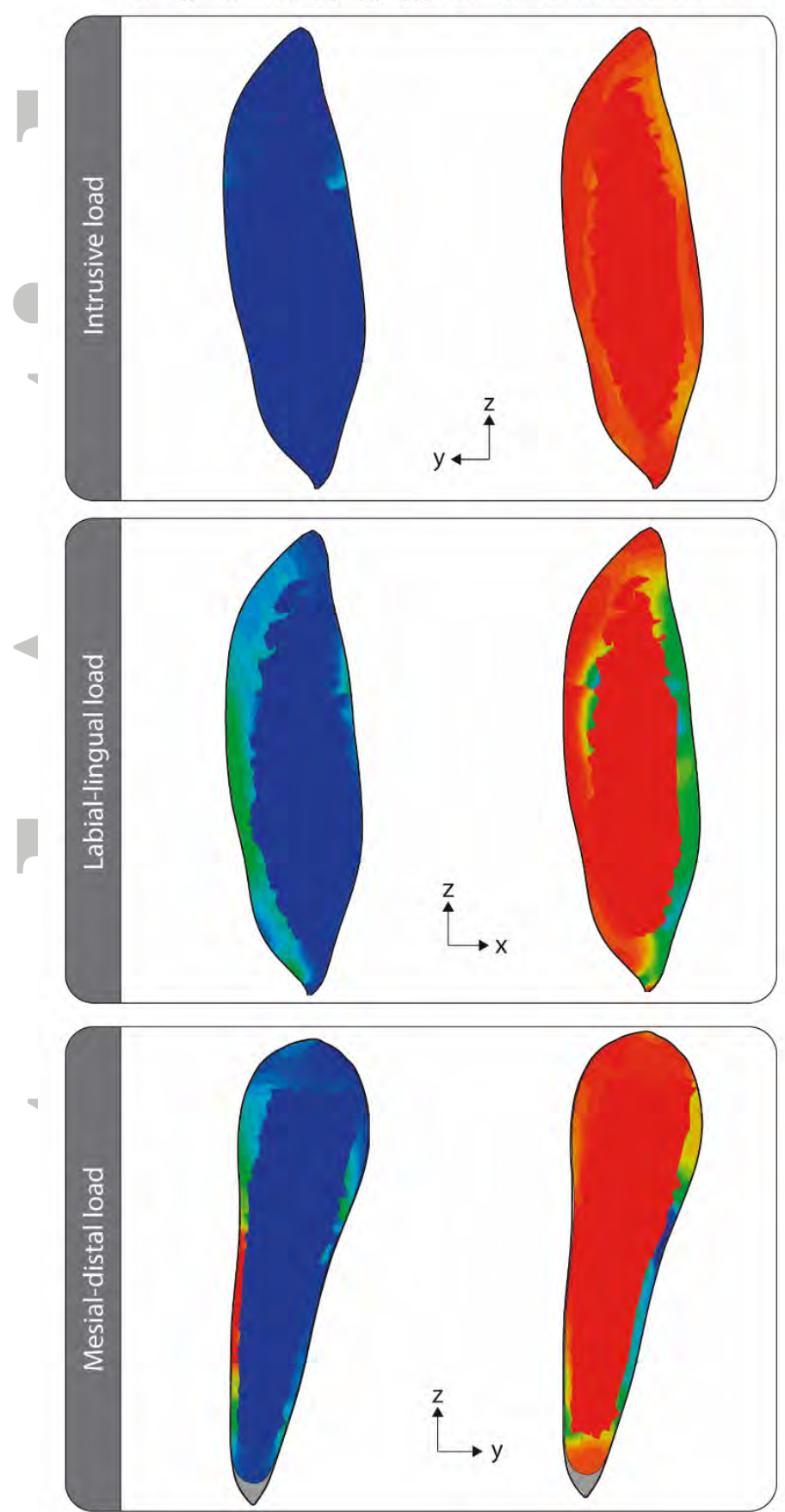

(iii)

Tooth displacement $(\mathrm{mm})$

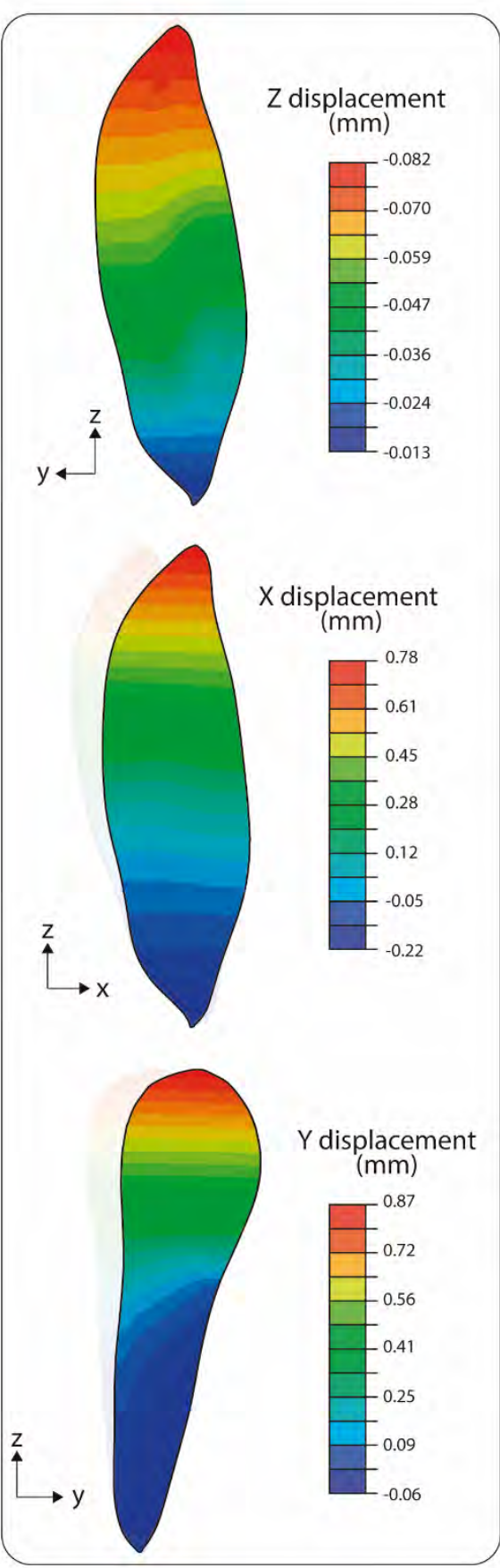

FIGURE A1 (i) Maximum principal stress, (ii) minimum principal stress and (iii) displacement of the canine tooth after applying a $360 \mathrm{~N}$ load at intrusive, labial-lingual and mesial-distal directions (ordered from top to bottom).

Tooth movement is directly related to the PDL stress distribution. Under intrusive loading, the liquid phase of the ligament partially reduces the minimum principal stress (Figure A2-ii) of the solid matrix of the PDL. Conversely, the porous pressure of the interstitial fluid increases (Figure A2-iii) and the volume of fluid decreases (Figure A2-iv) in the areas mainly compressed (Figure A2-ii). On the other hand, the apical fibres of the PDL try to support tooth rotation when lateral loads are applied. This role of the fibres increases the maximum 
principal stress of the solid matrix in the apical region (Figure A2-i). As shown in Figure 5C, over-elongation of the apical fibres leads to irreversible damage to the fibrous network in this area. Furthermore, the compressive force evacuates most of the fluid (Figure A2-iv), causing overpressure of the remaining interstitial fluid, mainly in the alveolar crest region (Figure A2iii).

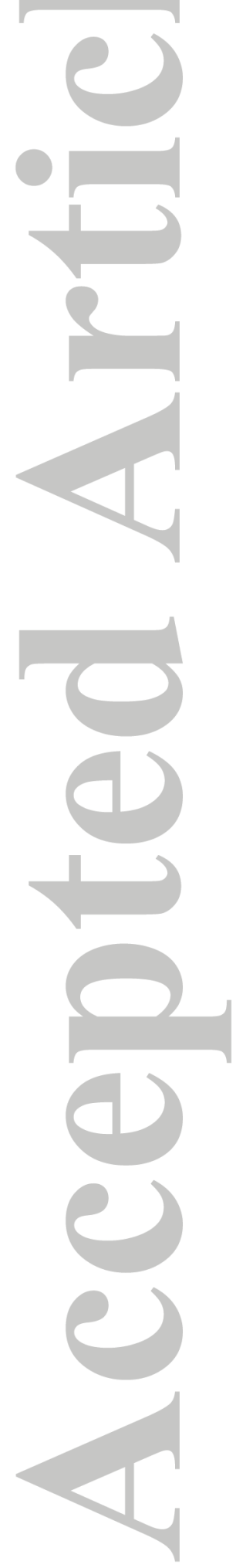


(i)

(ii)

Max. Principal Stress (MPa) Min. Principal Stress (MPa) $\mathrm{H}$

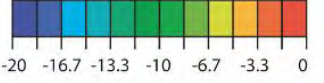

Porous Pressure $(\mathrm{kPa})$
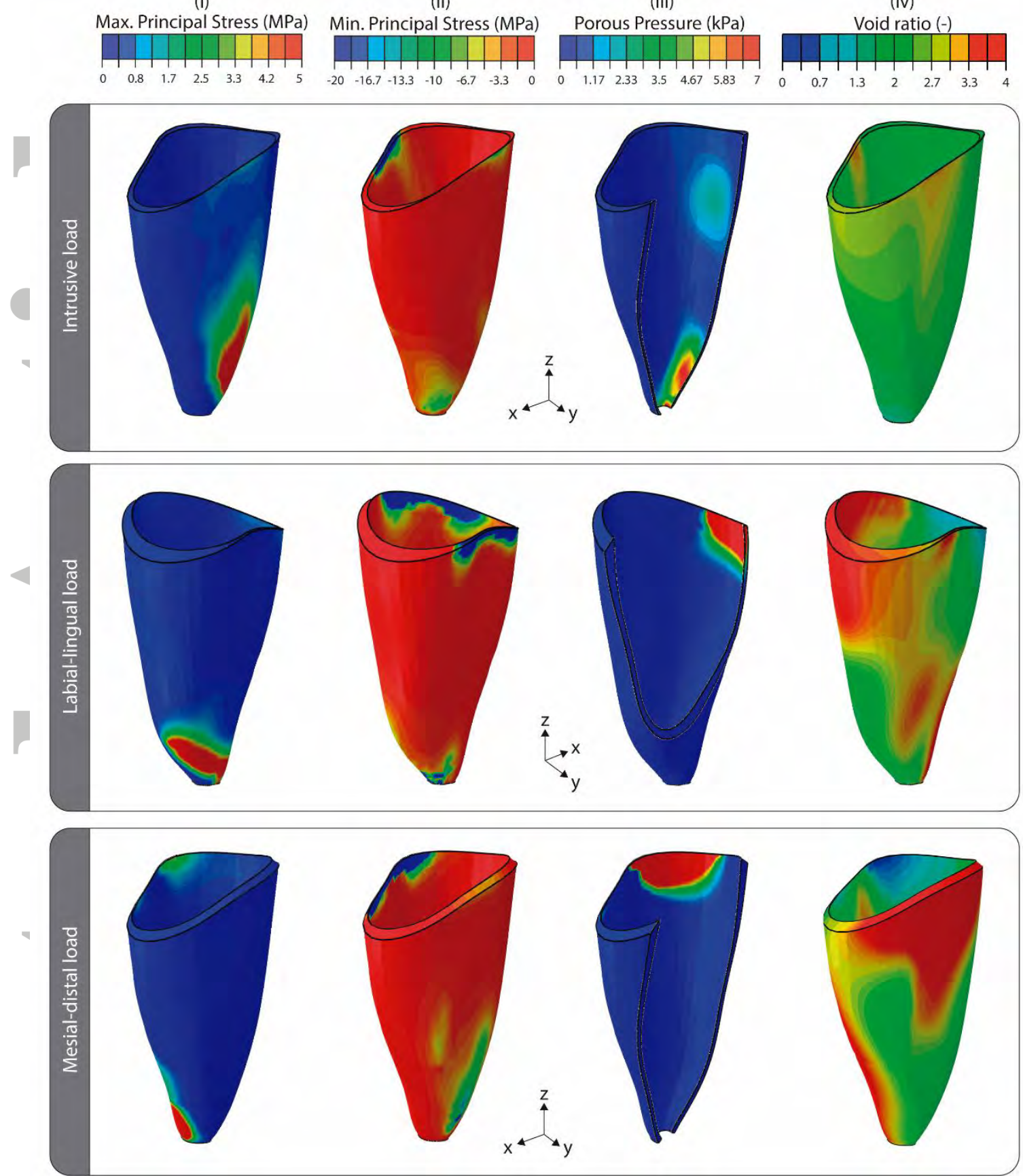

FIGURE A2 Distribution of (i) maximum principal stress, (ii) minimum principal stress, (iii) porous pressure and (iv) void ratio in the PDL after the first cycle of applying a $360 \mathrm{~N}$ load at intrusive, labial-lingual and mesial-distal directions (ordered from top to bottom).

As mentioned in the Results section, the apical region of the bone is mainly compressed when an intrusive load is applied (Figure A3). Moreover, the curved shape of the canine tooth compresses the bone at the middle and alveolar crest regions. On the other hand, lateral forces cause compressive stresses on two opposite areas of the apical and alveolar crest regions of the bone (Figure A3). The stress values on the bone are higher for lateral loads than for intrusive loads, especially in the layer of the apical region. 
(i)

Max. Principal Stress (MPa)

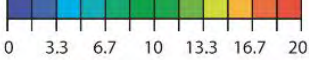

$\begin{array}{llllllll}0 & 3.3 & 6.7 & 10 & 13.3 & 16.7 & 20\end{array}$ (ii)

Min. Principal Stress (MPa)

$\begin{array}{llllllll}-20 & -16.7 & -13.3 & -10 & -6.7 & -3.3 & 0\end{array}$
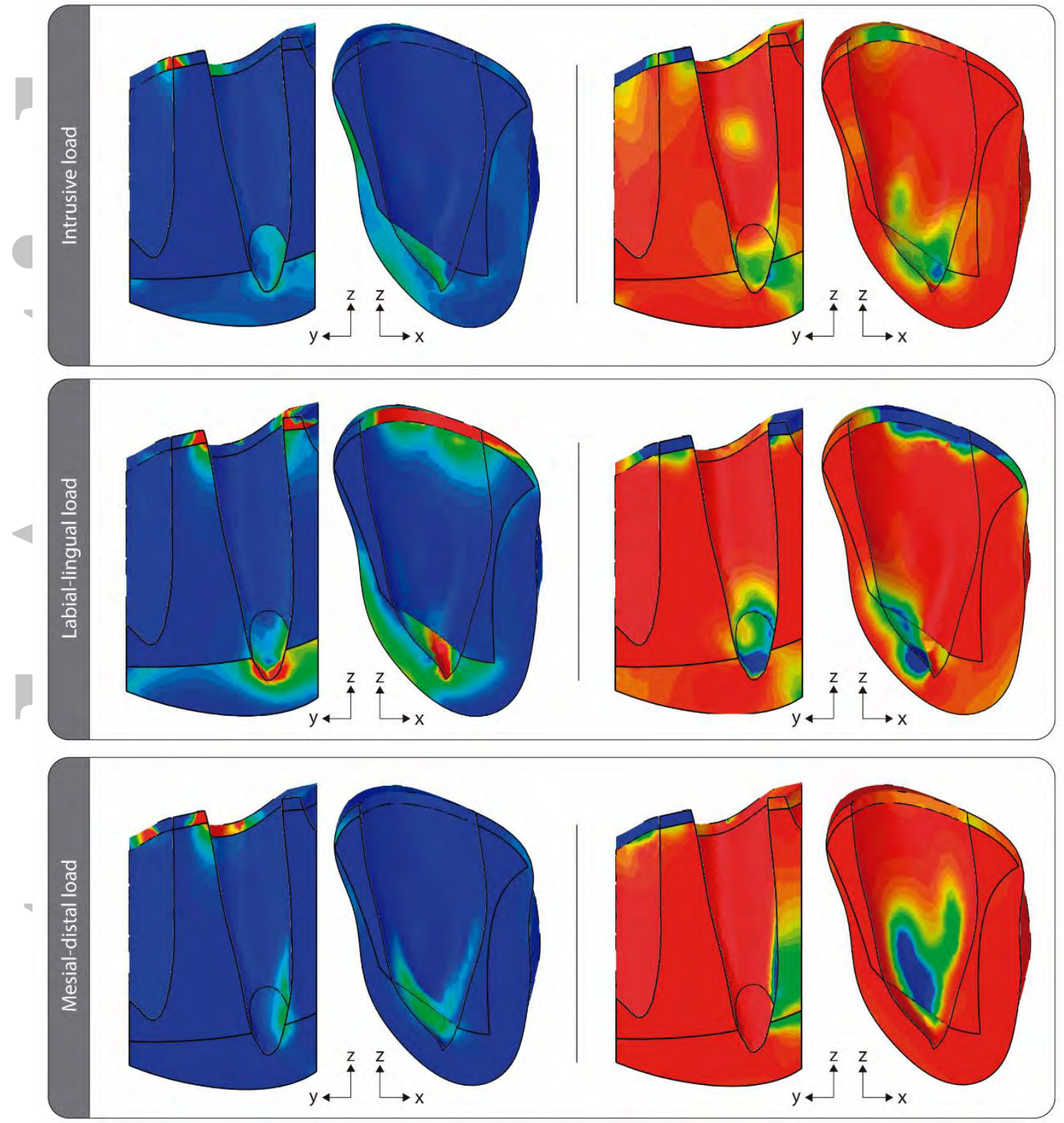

FIGURE A3 (i) Maximum and (ii) minimum principal stress distributions in the cortical and trabecular portions of the mandibular bone after the first cycle of applying a $360 \mathrm{~N}$ load at intrusive, labial-lingual and mesial-distal directions (ordered from top to bottom). 


\section{APPENDIX 2 - ALGORITHMIC PROCEDURE FOR IMPLEMENTATION OF THE POROUS FIBROUS HYPERELASTIC DAMAGE MATERIAL MODEL}

1. A database with the mechanical variables and $\left.D_{f}\right|^{k},\left.\eta\right|^{k}$ and $\left.\Xi_{t}^{m}\right|^{k}$ at $k$ time point is created.

2. The initial elastic stress tensor is computed.

3. The value of the fourth invariant $\widetilde{I_{4}}$ is obtained by Equation 2 .

3.1. If $\widetilde{I_{4}}<1$ :

$$
\Psi_{m}=\frac{2 \mu}{\alpha^{2}}\left[\hat{\lambda}_{1}^{\alpha}+\hat{\lambda}_{2}^{\alpha}+\hat{\lambda}_{3}^{\alpha}\right] \text { and } \Psi_{f}=0
$$

3.2. Otherwise, if $\widetilde{I_{4}} \geq 1$ :

$$
\Psi_{m}=C_{1} \cdot\left(\tilde{I}_{1}-3\right) \text { and } \Psi_{f}=\frac{k_{1}}{2 \cdot k_{2}}\left\{\exp \left[k_{2} \cdot\left(\tilde{I}_{4}-1\right)^{2}\right]-1\right\}
$$

3.2.1. The equivalent strain of the fibrous term is defined by Equation 14 .

3.2.2. The damage is checked:

3.2.2.1. If $\left.\Xi_{s}\right|^{k}>\left.\Xi_{t}^{m}\right|^{k}$, the $\left.D_{f}\right|^{k+1}$ parameter is computed by Equation 19 , and its value is updated.

3.2.2.2. Otherwise, if $\left.\Xi_{s}\right|^{k} \geq\left.\Xi_{t}^{m}\right|^{k}$, the damage is not updated $\left.D_{f}\right|^{k+1}=\left.D_{f}\right|^{k}$.

The damage variable $\eta$ and the continuous function $\Phi(\eta)$ are defined by Equations 12 and 13 .

4.1. If $\left.\psi_{\text {dev }}\right|^{k}>\left.\psi_{\text {dev }}^{m}\right|^{k}$ update $\left.\psi_{\text {dev }}^{m}\right|^{k+1}$ with $\left.\psi_{\text {dev }}\right|^{k}$ value.

4.2. Otherwise, if $\left.\psi_{d e v}\right|^{k} \leq\left.\psi_{d e v}^{m}\right|^{k}$, not update $\left.\psi_{d e v}^{m}\right|^{k}$ value.

5. The Cauchy stress tensor of the solid matrix is computed as $1 / J_{e l}$ times the push-forward of $\boldsymbol{S}^{86,87}$ by the following expression:

$$
\left.\overline{\boldsymbol{\sigma}}\right|^{k+1}=J^{-1} \chi_{*}(\boldsymbol{S})
$$

where the second Piola-Kirchhoff stress $\boldsymbol{S}$ tensor consists of the deviatoric and volumetric contributions ( $\boldsymbol{S}_{d e v}$ and $\boldsymbol{S}_{v o l}$, respectively) for the following strain energy density function:

$$
\begin{aligned}
\psi\left(\widetilde{\boldsymbol{C}}, \mathbf{M}, D_{f}, \eta\right)= & \psi_{d e v}\left(\widetilde{\boldsymbol{C}}, \mathbf{M}, D_{f}, \eta\right)+\psi_{v o l}\left(U_{e l}\right)=\psi_{d e v}\left(\widetilde{I_{1}}, \widetilde{I_{4}}, D_{f}, \eta\right)+\psi_{v o l}\left(U_{e l}\right) \\
& =\eta \psi_{m}\left(\widetilde{I_{1}}\right)+\eta\left(1-D_{f}\right) \psi_{f}\left(\widetilde{I_{4}}\right)+\psi_{v o l}\left(J_{e l}\right)+\Phi(\eta)
\end{aligned}
$$

Therefore:

$$
\boldsymbol{S}(\boldsymbol{C}, \mathbf{M})=2\left[\begin{array}{c}
\boldsymbol{S}(\boldsymbol{C}, \mathbf{M})=\boldsymbol{S}_{d e v}+\boldsymbol{S}_{v o l}=2\left[\frac{\partial \Psi_{d e v}}{\partial \boldsymbol{C}}+\frac{\partial \Psi_{v o l}}{\partial \boldsymbol{C}}\right] \\
\eta \frac{\partial \Psi_{m}\left(\widetilde{I_{1}}\right)}{\partial \widetilde{I_{1}}} \cdot \frac{\partial \widetilde{I_{1}}}{\partial \widetilde{\boldsymbol{C}}} \cdot \frac{\partial \widetilde{\boldsymbol{C}}}{\partial \boldsymbol{C}}+\Psi_{m} \frac{\partial \eta}{\partial \boldsymbol{C}}+\eta\left(1-D_{f}\right) \frac{\partial \Psi_{f}\left(\widetilde{I_{4}}\right)}{\partial \widetilde{I_{4}}} \cdot \frac{\partial \widetilde{I_{4}}}{\partial \widetilde{\boldsymbol{C}}} \cdot \frac{\partial \widetilde{\boldsymbol{C}}}{\partial \boldsymbol{C}}+ \\
\left(1-D_{f}\right) \psi_{f} \frac{\partial \eta}{\partial \boldsymbol{C}}-\eta \Psi_{f} \frac{\partial D_{f}}{\partial \boldsymbol{C}}+\frac{\Psi_{v o l}\left(J_{e l}\right)}{\partial J_{e l}} \frac{\partial J_{e l}}{\partial \boldsymbol{C}}+\frac{\partial \Phi}{\partial \eta} \frac{\partial \eta}{\partial \boldsymbol{C}}
\end{array}\right]
$$

6. The total stress, $\boldsymbol{\sigma}$, in the fully saturated tissue with the porous contribution coupled is defined by Equation 9.

7. The initial elastic modulus ${ }^{86,87}$ is calculated as $1 / J_{e l}$ times the push-forward of the elasticity tensor $\mathbf{C}$ by:

$$
\mathbf{c}^{k+1}=J^{-1} \chi_{*}(\mathbf{C})
$$

where the elasticity tensor is obtained from the second Piola-Kirchhoff stress tensor by:

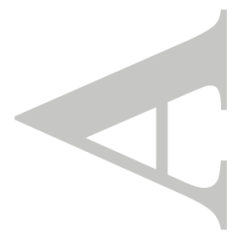

$$
\mathbf{C}=\mathbf{C}_{d e s}+\mathbf{C}_{v o l}=2 \frac{\partial \boldsymbol{S}(\boldsymbol{C}, \mathbf{M})}{\partial \boldsymbol{C}}=4\left[\frac{\partial^{2} \Psi_{d e v}(\boldsymbol{C}, \mathbf{M})}{\partial \boldsymbol{C} \otimes \partial \boldsymbol{C}}+\frac{\partial^{2} \psi_{v o l}\left(U_{e l}\right)}{\partial \boldsymbol{C} \otimes \partial \boldsymbol{C}}\right]
$$

